$$
\begin{aligned}
& 1 N-07 \\
& 6277
\end{aligned}
$$

NASA Technical Memorandum 107103

AIAA-96-0449

\title{
Rapid Numerical Simulation of Viscous Axisymmetric Flow Fields
}

Daniel L. Tweedt and Rodrick V. Chima

Lewis Research Center

Cleveland, Ohio

Prepared for the

34th Aerospace Sciences Meeting and Exhibit

sponsored by the American Institute of Aeronautics and Astronautics Reno, Nevada, January 15-18, 1996

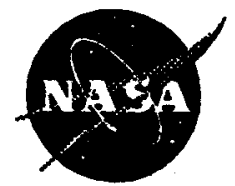

(NASA-TM-107103) RAPID NUMERICAL 


\title{
Rapid Numerical Simulation of Viscous Axisymmetric Flow Fields
}

\author{
Daniel L. Tweedt ${ }^{\dagger}$ and Rodrick V. Chima ${ }^{\ddagger}$ \\ NASA Lewis Research Center \\ Cleveland, Ohio 44135
}

\begin{abstract}
A two-dimensional Navier-Stokes code has been developed for rapid numerical simulation of axisymmetric flow fields, including flow fields with an azimuthal velocity component. The azimuthal-invariant Navier-Stokes equations in a cylindrical coordinate system are mapped to a general body-fitted coordinate system, with the streamwise viscous terms then neglected by applying the thinlayer approximation. Turbulence effects are modeled using an algebraic model, typically the Baldwin-Lomax turbulence model, although a modified Cebeci-Smith model can also be used. The equations are discretized using central finite differences and solved using a multistage Runge-Kutta algorithm with a spatially-varying time step and implicit residual smoothing.

Results are presented for calculations of supersonic flow over a waisted body-of-revolution, transonic flow through a normal shock wave in a straight circular duct of constant cross-sectional area, swirling supersonic (inviscid) flow through a strong shock in a straight radial duct, and swirling subsonic flow in an annular-to-circular diffuser duct. Comparisons between computed and experimental results are in fair to good agreement, demonstrating that the viscous code can be a useful tool for practical engineering design and analysis work.
\end{abstract}

\section{Introduction}

Axisymmetric flow fields are frequently encountered in internal and external aerodynamics, within various engineering research areas such as ballistics, aircraft inlets and nozzles, and circular/annular internal duct flows. Often these flows involve velocities in the azimuthal direction. For the rapid simulation of such flow fields, a computer code has been developed which solves the thin-

\footnotetext{
${ }^{\dagger}$ Aerospace Engineer

${ }^{\ddagger}$ Aerospace Engineer, Associate Fellow AIAA
}

layer formulation of the azimuthal-invariant NavierStokes equations, including the momentum equation in the azimuthal direction.

The original work in developing and computationally applying an azimuthal-invariant thin-layer form of the Navier-Stokes equations, including the momentum equation for the azimuthal direction, was done by Nietubicz et al. [1-2]. In that work, the three-dimensional, transformed, thin-layer equations in Cartesian coordinates were reduced to an azimuthal-invariant set by an appropriate coordinate selection and subsequent integration in the azimuthal direction to determine axisymmetric source terms. Since the thin-layer approximation was applied before reducing the equations, only the inviscid source terms were determined. In the present work the thin-layer approximation has been applied after transformation of the axisymmetric Navier-Stokes equations to the computational domain, so that viscous source terms were retained in the equation set. At moderate to high Reynolds numbers, however, where the thin-layer approximation is valid, these viscous sources are probably always small and could be neglected. Nevertheless, they have not been discarded since their retention has little impact on computational efficiency.

The numerical solution of the governing equations is performed using an explicit time-marching scheme with central finite differences for spatial discretization. The time marching is carried out to steady-state conditions, with the convergence rate accelerated using a spatiallyvarying time step and implicit residual smoothing. The baseline code is robust, executes rapidly, and vectorizes well on vector processors.

Several flow field solutions are presented that demonstrate the application, accuracy, and practical usefulness of the method. Specifically, results are presented for calculations of supersonic flow over a waisted body-of-revolution, transonic flow through a normal shock wave in a straight circular duct of constant cross-sectional area, swirling supersonic (inviscid) flow through a strong shock in a straight radial duct, and swirling subsonic flow in an annular-to-circular diffuser duct. Experimental or analytical data are compared to the computed results, except in the last test case, with generally fair to good agreement between the two. 


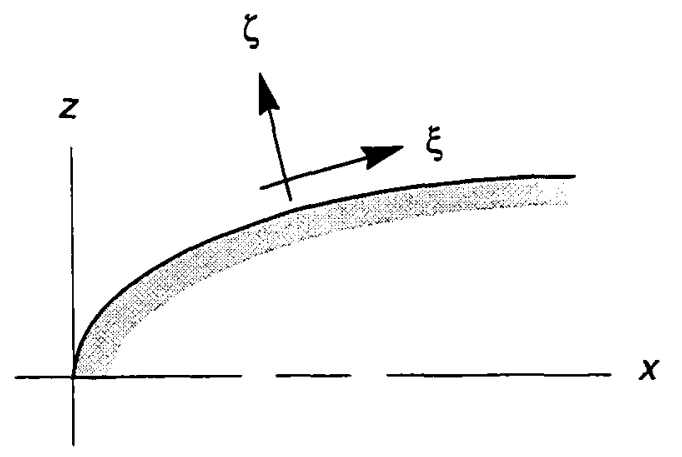

Figure 1- Axisymmetric coordinate system

\section{Governing Equations}

The Navier-Stokes equations are written in a stationary cylindrical coordinate system, and all terms with derivatives in the azimuthal-direction are excluded. The cylindrical equations are mapped to a general body-fitted $(\xi, \zeta)$ coordinate system using standard techniques. As illustrated in Figure 1, the $\xi$-coordinate direction is along the body-surface, and therefore approximately along the flow direction, while the $\zeta$-direction is roughly normal to the flow. The viscous derivatives in the $\xi$-direction are dropped by applying the thin-layer approximation [1-3]. Using $(x, y, z)$ for denoting the cylindrical coordinate directions, with $x$ in the axial direction, $y$ in the azimuthal direction, and $z$ in the radial direction, the resulting equations can be written as follows:

$$
\partial_{t} \hat{q}+\partial_{\xi} \hat{E}+\partial_{\zeta}\left(\hat{G}-R e^{-1} \hat{S}\right)=\hat{H}+R e^{-1} \hat{K}
$$

where

$$
\begin{gathered}
\hat{q}=J^{-1}[\rho, \rho u, \rho v, \rho w, e]^{T} \\
\hat{E}=J^{-1}\left[\begin{array}{c}
\rho U \\
\rho u U+\xi_{x} p \\
\rho v U \\
\rho w U+\xi_{z} p \\
(e+p) U
\end{array}\right] \quad \hat{G}=J^{-1}\left[\begin{array}{c}
\rho W \\
\rho u W+\zeta_{x} p \\
\rho v W \\
\rho w W+\zeta_{z} p \\
(e+p) W
\end{array}\right] \\
\hat{S}=J^{-1}\left[\begin{array}{c}
0 \\
S_{2} \\
S_{3} \\
S_{4} \\
S_{5}
\end{array}\right] \quad \hat{H}=\frac{J^{-1}}{z}\left[\begin{array}{c}
0 \\
0 \\
-\rho v w \\
\rho v^{2}+p \\
0
\end{array}\right] \quad \hat{K}=\frac{J^{-1}}{z}\left[\begin{array}{c}
0 \\
0 \\
K_{3} \\
K_{4} \\
0
\end{array}\right]
\end{gathered}
$$

The absolute velocity components $u, v$, and $w$ point in the $x, y$, and $z$ coordinate directions, respectively. The contravariant velocity components are given by

$$
\begin{aligned}
& U=\xi_{x} u+\xi_{z} w \\
& W=\zeta_{x} u+\zeta_{z} w
\end{aligned}
$$

Assuming an ideal gas with constant specific heats, then $\gamma$ (which is $c_{p} / c_{v}$ ) is constant, and the energy and static pressure are given by

$$
\begin{aligned}
& e=\rho\left[\frac{a^{2}}{\gamma(\gamma-1)}+\frac{1}{2}\left(u^{2}+v^{2}+w^{2}\right)\right] \\
& p=(\gamma-1)\left[e-\frac{1}{2} \rho\left(u^{2}+v^{2}+w^{2}\right)\right]
\end{aligned}
$$

where the sonic velocity $a$ is related to static pressure and density by the equation of state:

$$
a^{2}=\frac{\gamma p}{\rho}
$$

The terms in the viscous flux vector $\hat{S}$ can be written as follows:

$$
\begin{aligned}
& S_{2}=\zeta_{x} \sigma_{x x}+\zeta_{z} \sigma_{x z} \\
& S_{3}=\mu\left(\zeta_{x}^{2}+\zeta_{z}^{2}\right) v_{\zeta}-\zeta_{z} \frac{v}{z} \\
& S_{4}=\zeta_{x} \sigma_{x z}+\zeta_{z} \sigma_{z z} \\
& S_{5}=\frac{\left(\zeta_{x}^{2}+\zeta_{z}^{2}\right) \kappa}{(\gamma-1) P r}\left(a^{2}\right)_{\zeta}+S_{2} u+S_{3} v+S_{4} w
\end{aligned}
$$

where the stress terms are

$$
\begin{aligned}
& \sigma_{x x}=2 \mu \zeta_{x} u_{\zeta}+\lambda \nabla \cdot V \\
& \sigma_{x z}=\mu\left(\zeta_{z} u_{\zeta}+\zeta_{x} w_{\zeta}\right) \\
& \sigma_{z z}=2 \mu \zeta_{z} w_{\zeta}+\lambda \nabla \cdot V
\end{aligned}
$$

The terms in the viscous source vector $\hat{K}$ are given by

$$
\begin{aligned}
& K_{3}=\mu\left(\zeta_{z} \nu_{\zeta}-\frac{v}{z}\right) \\
& K_{4}=-\frac{2 \mu w}{z}-\lambda \nabla \cdot V
\end{aligned}
$$

Stokes' hypothesis, $\lambda=-2 \mu / 3$, is assumed, and the dynamic viscosity $\mu$ and the thermal conductivity $K$ are dimensionless. The velocity divergence $\nabla \cdot V$ in the dilatation term is given by

$$
\nabla \cdot v=\zeta_{x} u_{\zeta}+\zeta_{z} w_{\zeta}+\frac{w}{z}
$$


The inverse-Jacobian $J^{-1}$ is given by the relation

$$
J^{-1}=\frac{1}{J}=z\left(x_{\xi} z_{\zeta}-x_{\zeta} z_{\xi}\right)
$$

with the metric terms defined as follows:

$$
\left[\begin{array}{l}
\xi_{x} \\
\xi_{z} \\
\zeta_{x} \\
\zeta_{z}
\end{array}\right]=J\left[\begin{array}{c}
z z_{\zeta} \\
-z x_{\zeta} \\
-z z_{\xi} \\
z x_{\xi}
\end{array}\right]
$$

The metric terms and Jacobian are calculated from the grid coordinates by using second-order central differences. In order for the metrics to be geometrically consistent with the Viviand strong conservation form of the governing equations [4], the following constraints must be met:

$$
\begin{aligned}
\partial_{\xi} x_{\zeta} & =\partial_{\zeta} x_{\xi} \\
\partial_{\xi}\left(z^{2}\right)_{\zeta} & =\partial_{\zeta}\left(z^{2}\right)_{\xi}
\end{aligned}
$$

These constraints can be satisfied in the discretized metrics by using the following difference equations:

$$
\begin{aligned}
\xi_{x} & =\frac{J}{4}\left(z_{i, j+1}+z_{i, j-1}\right)\left(z_{i, j+1}-z_{i, j-1}\right) \\
-\xi_{z} & =\frac{J}{4}\left(z_{i, j+1}+z_{i, j-1}\right)\left(x_{i, j+1}-x_{i, j-1}\right) \\
-\zeta_{x} & =\frac{J}{4}\left(z_{i+1, j}+z_{i-1, j}\right)\left(z_{i+1, j}-z_{i-1, j}\right) \\
\zeta_{z} & =\frac{J}{4}\left(z_{i+1, j}+z_{i-1, j}\right)\left(x_{i+1, j}-x_{i-1, j}\right)
\end{aligned}
$$

where the $i$ - and $j$-indices correspond to the $\xi$ - and $\zeta$-directions, respectively. In order to avoid numerical sources, the inverse-Jacobian is calculated using a rewritten form of Equation (10):

$$
J^{-1}=z\left[\partial_{\xi}\left(-z x_{\zeta}\right)+\partial_{\zeta}\left(z x_{\xi}\right)\right]
$$

with the radius $z$ outside the brackets taken at the central node, and central differences applied to the discretized form of the terms in parenthesis:

$$
\begin{aligned}
-z x_{\zeta} & =\frac{1}{4}\left(z_{i, j+1}+z_{i, j-1}\right)\left(x_{i, j+1}-x_{i, j-1}\right) \\
z x_{\xi} & =\frac{1}{4}\left(z_{i+1, j}+z_{i-1, j}\right)\left(x_{i+1, j}-x_{i-1, j}\right)
\end{aligned}
$$

The equations are nondimensionalized by constant reference properties; namely, density $\rho_{\text {ref }}$, sonic velocity $a_{r e f}$, and dynamic viscosity $\mu_{\text {ref }}$, all typically based on inlet total conditions. The Reynolds number $R e$ and Prandtl number $P r$ are defined in terms of these properties.

For turbulent flows the dimensionless dynamic viscosity $\mu$ is determined by

$$
\mu=\mu_{l a m}+\mu_{t u r b}
$$

where the laminar viscosity is calculated using a powerlaw function of dimensionless temperature $T$ :

$$
\mu_{\text {lam }}=((\gamma-1) T)^{n}=\left(a^{2}\right)^{n}
$$

with $n \approx 2 / 3$ for air over a wide range of temperatures. The turbulent eddy-viscosity $\mu_{t u r b}$ is calculated using an algebraic turbulence model, either the Baldwin-Lomax model [5] or a modified version of the Cebeci-Smith model [6]. The vorticity magnitude used in the turbulence model is calculated according to the following relation:

$$
|\omega|=\sqrt{\omega_{x}^{2}+\omega_{y}^{2}+\omega_{z}^{2}}
$$

where

$$
\begin{aligned}
\omega_{x} & =\xi_{z} v_{\xi}+\zeta_{z} v_{\zeta}+\frac{v}{z} \\
\omega_{y} & =\xi_{x} w_{\xi}+\zeta_{x} w_{\zeta}-\xi_{z} u_{\xi}-\zeta_{z} u_{\zeta} \\
-\omega_{z} & =\xi_{x} v_{\xi}+\zeta_{x} v_{\zeta}
\end{aligned}
$$

The dimensionless thermal conductivity term, $\kappa$ over Prandtl number $\mathrm{Pr}$, is determined as follows for turbulent flows:

$$
\frac{\kappa}{P r}=\frac{\mu_{\text {lam }}}{P r_{\text {lam }}}+\frac{\mu_{\text {turb }}}{P r_{\text {turb }}}
$$

with the turbulent Prandtl number assumed equal to 0.90 .

The axisymmetric governing equations can be reduced to the planar two-dimensional set by eliminating the source terms $\hat{H}$ and $\hat{K}$ on the right-hand-side of Equation (1), setting the azimuthal velocity $v$ to zero everywhere, setting the (undifferentiated) radius $z$ equal to one in Equations (10) and (11), and eliminating the term $w / z$ in Equation (9).

\section{Boundary Conditions}

Different boundary types can apply to a particular problem depending on the specified geometry and whether the problem involves an internal or external flow field. The boundary types addressed below include the axis-ofsymmetry, the solid wall with and without the no-slip condition, and the inflow and outflow boundaries for internal flow fields. Boundary conditions suited mostly for external flow fields are not addressed.

At the inflow boundary the total temperature, total pressure, and whirl ( $v$-velocity) are fixed according to the 
specified inflow condition. For flows with subsonic (throughflow) velocities at the inflow boundary, a steady characteristic relation using the upstream-running Riemann invariant is used to solve for the remaining quantities at the boundary. The Riemann invariant is based on the meridional velocity component, and the meridional flow angles are fixed at the specified input values [7-9]. Since in internal flows the inflow boundary can involve large radial velocities and viscous shear layers at walls, a non-isentropic formulation of the steady, axisymmetric characteristic equation is applied. Assuming that the inflow direction is nearly parallel to the $\xi$-direction, the steady axisymmetric characteristic equation can be written as follows:

$$
\partial_{\xi} R^{-}=\frac{\left(v^{2}+a V_{m}\right)\left(\frac{\partial_{\xi} z}{z}\right)+\Gamma}{V_{m}-a}
$$

where

$$
\Gamma=\left(\frac{a^{2}}{\gamma}-a V_{m}\right)\left(\frac{\gamma}{\gamma-1} \frac{\partial_{\xi} T_{t}}{T_{t}}-\frac{\partial_{\xi} p_{t}}{p_{t}}\right)
$$

The Riemann invariant based on meridional velocity is given by

$$
R^{-}=V_{m}-\frac{2 a}{\gamma-1}
$$

where

$$
V_{m}=\sqrt{u^{2}+w^{2}}
$$

The flow variables on the right-hand-side of Equation (20) are taken from the interior, and all derivatives are obtained by first-order (backward) differences from boundary to interior. The resulting formulation is solved for $R^{-}$at the inlet boundary. Note that the entropy-gradient term $\Gamma$ is non-zero only within viscous regions. The meridional velocity at the inlet is calculated from the total temperature and the Riemann-invariant by using the following equation:

$$
V_{m}=\frac{(\gamma-1) R^{-}+\sqrt{B-2 C}}{\gamma+1}
$$

where

$$
\begin{aligned}
& B=4 \frac{(\gamma+1)}{(\gamma-1)} a_{t}^{2} \\
& C=(\gamma+1) v^{2}+(\gamma-1)\left(R^{-}\right)^{2}
\end{aligned}
$$

For convenience, the term $B$ uses the dimensionless total sonic velocity rather than the total temperature. Once the meridional velocity is known, the velocity components $u$ and $w$ can be algebraically determined using the specified meridional flow angle. Finally, using isentropic flow rela- tions the density is calculated from the total velocity magnitude and the specified total temperature and total pressure.

For supersonic inflow all flow quantities at the inlet boundary are fixed.

At the outflow boundary the conservation variables $\rho$, $\rho u, \rho v$, and $\rho w$ are extrapolated from the interior. For flow fields with supersonic outflow the energy conservation variable $e$ is extrapolated as well. For flow fields with subsonic outflow the static pressure $p$ is specified at the outer radial position, and the remaining local static pressures along the exit boundary are calculated by integrating the simple radial-equilibrium equation:

$$
\frac{d p}{d z}=\frac{\rho v^{2}}{z}
$$

Along solid walls with a slip-condition (for inviscid computations) the flow is obtained by linear extrapolation of the density $\rho$, the static pressure $p$, the azimuthal velocity $\nu$, and the covariant velocity $U^{\prime}$ given by

$$
U^{\prime}=\frac{x_{\xi} u+z_{\xi} w}{\sqrt{x_{\xi}^{2}+z_{\xi}^{2}}}=\frac{\zeta_{z} u-\zeta_{x} w}{\sqrt{\zeta_{x}^{2}+\zeta_{z}^{2}}}
$$

The wall tangency condition is satisfied by setting the contravariant velocity $W$ equal to zero.

The axis-of-symmetry boundary condition is similar to the slip-wall condition except that the radial velocity $w$ is identically zero. The azimuthal velocity $v$ is also zero for viscous computations, while for inviscid computations it is linearly extrapolated. The axial velocity $u$ and static temperature $T$ are determined using second-order onesided differences such that $u_{\zeta}=T_{\zeta}=0$. The static pressure is linearly extrapolated, and the density follows from the equation of state. Note that for inviscid computations the axis flow quantities are determined only for aesthetic reasons since they do not influence the flow field solution.

For solid walls with the no-slip condition (for viscous computations) the velocities $u$ and $w$ are zero, and $v=\Omega z$, where $\Omega$ is the angular velocity of the wall. The wall static pressure is obtained by zero-order extrapolation from the adjacent interior point; that is, $p_{\zeta}=0$. The wall static temperature is either specified on input, or else determined assuming an adiabatic wall, $T_{\zeta}=0$, and using a second-order one-sided difference.

\section{Multistage Runge-Kutta Scheme}

The governing equations are discretized using a nodecentered finite-difference scheme, with second-order central differences used throughout. The multistage RungeKutta scheme developed by Jameson, Schmidt, and Turkel $[10]$ is used to advance the flow equations in time from an 
initial guess to a steady state. Equation (1) can be rewritten in the following terse form:

$$
\partial_{t} q=-J\left[R_{I}-\left(R_{V}+D\right)\right]
$$

where $R_{I}$ is the inviscid residual including source term, $R_{V}$ is the viscous residual including source term, and $D$ is the artificial dissipation term described in the next subsection. This allows the multistage Runge-Kutta algorithm to be expressed as follows:

$$
\begin{aligned}
q_{0} & =q_{n} \\
q_{1} & =q_{0}-\alpha_{1} J \Delta t\left[R_{I} q_{0}-\left(R_{V}+D\right) q_{0}\right] \\
& \cdot \\
& \cdot \\
& \cdot \\
q_{k} & =q_{0}-\alpha_{k} J \Delta t\left[R_{I} q_{k-1}-\left(R_{V}+D\right) q_{0}\right] \\
q_{n+1} & =q_{k}
\end{aligned}
$$

In most applications a standard four-stage scheme is used, with

$$
\alpha_{k}=[1 / 4,1 / 3,1 / 2,1]
$$

For efficiency both the physical and artificial dissipation terms are calculated only at the first stage and then held constant for subsequent stages.

\section{Artificial Dissipation}

The dissipative term $D$ in Equation (26) is similar to that used by Jameson et al. [10]. It is given by

$$
D q=\left(D_{\xi}+D_{\zeta}\right) q
$$

where the $\xi$-direction operator is given by

$$
D_{\xi} q=\partial_{\xi}\left[J^{-1} C_{\xi}\left(V_{2} \partial_{\xi} q+V_{4} \partial_{\xi \xi \xi} q\right)\right]
$$

The term $C_{\xi}$ is a coefficient, and the terms $V_{2}$ and $V_{4}$ are the following:

$$
\begin{aligned}
v_{2} & =\mu_{2} \max \left(v_{i-1}, v_{i}, v_{i+1}, v_{i+2}\right) \\
v_{4} & =\max \left(0, \mu_{4}-v_{2}\right)
\end{aligned}
$$

where

$$
\mathrm{v}_{i}=\frac{\left|p_{i+1}-2 p_{i}+p_{i-1}\right|}{\min \left(p_{n},\left|p_{i+1}+2 p_{i}+p_{i-1}\right|\right)}
$$

and the $i$-indexing corresponds to the $\xi$-direction. The constant $\mu_{2}$ scales a first-order artificial viscosity that is switched on at shocks detected by Equation (31). The denominator in Equation (31) is normally constant at the pressure $p_{n}$, making the operator roughly symmetric across shocks. For external flows $p_{n}$ is equal to the static pressure at infinity, and for internal flows it is normally a fixed low pressure at either the grid inlet or exit. The more commonly applied term $\left|p_{i+1}+2 p_{i}+p_{i-1}\right|$ is included to switch on the second-difference dissipation when the pressure becomes very small, usually due to numerical problems. The constant $\mu_{4}$ scales a uniform third-order artificial viscosity that is switched off at shocks by Equation (31). Typically, values for $\mu_{2}$ and $\mu_{4}$ are $1 / 8$ and $1 / 64$, respectively.

The coefficient $C_{\xi}$ is arbitrary, but can have a large impact on stability and solution accuracy. It has been found in general that directionally-biased coefficients work substantially better than directionally-independent ones. A directionally-biased coefficient which works well is similar to one proposed by Kunz and Lakshminarayana [11], which was a modification of an earlier one proposed by Martinelli and Jameson [12]. For the two-dimensional axisymmetric case it can be written as follows for each grid direction:

$$
\begin{aligned}
& c_{\xi}=\left(\Delta t_{\xi}^{-1}+\Delta t_{a}^{-1}\right) \sqrt{1+\frac{\Delta t_{\zeta}^{-1}}{\Delta t_{\xi}^{-1}+\Delta t_{a}^{-1}}} \\
& c_{\zeta}=\left(\Delta t_{\zeta}^{-1}+\Delta t_{a}^{-1}\right) \sqrt{1+\frac{\Delta t_{\xi}^{-1}}{\Delta t_{\zeta}^{-1}+\Delta t_{a}^{-1}}}
\end{aligned}
$$

To minimize artificial dissipation in viscous regions, the coefficients in the $\zeta$-direction are reduced to zero quadratically over several grid points near walls. The onedimensional time steps in Equations (32) are given below in connection with the stability limit.

\section{Stability Limit}

The following expression, obtained in part through a linear stability analysis, and in part by transforming threedimensional Cartesian linear stability results $[8,9,13]$ to an axisymmetric coordinate system, is used for the time step:

$$
\Delta t \leq \frac{\lambda^{*}}{\Delta t_{\xi}^{-1}+\Delta t_{\zeta}^{-1}+\Delta t_{a}^{-1}+\Delta t_{v}^{-1}}
$$

where $\lambda^{*}$ is the maximum Courant number for the particular multistage scheme, which for the standard four-stage scheme is approximately 2.8 . The inverse one-dimensional time steps are given by 


$$
\begin{aligned}
& \Delta t_{\xi}^{-1}=\left|\xi_{x} u\right|+\left|\xi_{z} w\right|+a \sqrt{\xi_{x}^{2}+\xi_{z}^{2}} \\
& \Delta t_{\zeta}^{-1}=\left|\zeta_{x} u\right|+\left|\zeta_{z} w\right|+a \sqrt{\zeta_{x}^{2}+\zeta_{z}^{2}} \\
& \Delta t_{a}^{-1}=\frac{\gamma|w|}{z} \\
& \Delta t_{v}^{-1}=k_{t} \frac{\gamma \mu}{\rho P r}\left(\xi_{x}^{2}+\xi_{z}^{2}+\zeta_{x}^{2}+\zeta_{z}^{2}+\psi\right)
\end{aligned}
$$

In the viscous time-step contribution $\Delta t_{v}^{-1}$, the constant $k_{t}$ is given a value of 4.0 , and an axisymmetric term $\Psi$ is included where

$$
\psi=\left(\frac{J^{-1}}{z}\right)^{2} \frac{\left(\xi_{x}^{2}+\xi_{z}^{2}\right)\left(\zeta_{x}^{2}+\zeta_{z}^{2}\right)}{z^{2}}
$$

To accelerate convergence to a steady state, the maximum permissible time step at each grid point is used giving a constant Courant number everywhere. The time step is normally updated every 10 iterations.

\section{Implicit Residual Smoothing}

To further accelerate convergence it is desirable to use a time step even larger than the stability limit given by Equation (33). To maintain stability, the residual calculated in Equation (26) is smoothed after each Runge-Kutta stage by an implicit smoothing operator:

$$
\left(1-\varepsilon_{\xi} \delta_{\xi \xi}\right)\left(1-\varepsilon_{\zeta} \delta_{\zeta \zeta}\right) R_{k}=R_{k}
$$

where $\delta_{\xi \xi}$ and $\delta_{\zeta \zeta}$ are standard second difference operators, and $\varepsilon_{\xi}$ and $\varepsilon_{\zeta}$ are smoothing parameters.

Linear stability analysis shows that the Runge-Kutta scheme may be made unconditionally stable using implicit residual smoothing if the smoothing parameters $\varepsilon$ are made sufficiently large [14]. In one dimension

$$
\varepsilon \geq \frac{1}{4}\left[\left(\frac{\lambda}{\lambda^{*}}\right)^{2}-1\right]
$$

gives unconditional stability if $\lambda^{*}$ is the Courant limit of the unsmoothed scheme, and $\lambda$ is a larger operating Courant number. In two dimensions different $\varepsilon$ 's may be used in each direction, and their magnitudes may often be reduced below the value given by Equation (37). Good success has been achieved by using the artificial dissipation coefficients $C_{\xi}$ and $C_{\zeta}$ in Equations (32) to scale $\varepsilon$ in the two directions:

$$
\begin{aligned}
\varepsilon_{\xi} & =C_{\xi} \varepsilon \\
\varepsilon_{\zeta} & =C_{\zeta} \varepsilon
\end{aligned}
$$

which is an approach similar to that of Martinelli and Jameson [12]. Typically, the value for $\varepsilon$ can be taken as the minimum limit from Equation (37), which usually works well for Courant numbers $\lambda$ up to 7.0.

\section{Computational Details}

The code used to simulate the flow fields discussed below is referred to as DVC2D (Duct Viscous Code 2D), and it was written primarily for internal flow fields. The code can be run as an inviscid (Euler) solver, or as a viscous solver with or without turbulence modeling. In all viscous cases presented below, the Baldwin-Lomax turbulence model was used and updated every five iterations.

Computational grids were generated using an interactive program called TIGGERC [15] in conjunction with interior grid restretching using an elliptic partial differential equation (PDE) solver [16]. The elliptic PDE solver forces grid orthogonality at solid boundaries, and allows user control of the grid spacing normal and adjacent to the boundaries, and of the stretching away from the boundaries.

The results discussed in Reference 6, along with some recent computational studies done by the authors, reveal that an adjustment to the Baldwin-Lomax turbulence model parameters $C_{K l e b}$ and $C_{c p}$ gives better solutions for turbulent flat-plate boundary layers. The suggested parameter values are

$$
\begin{aligned}
& C_{\text {Kleb }}=0.646 \\
& C_{c p}=\max \left(1.216,0.8 M_{e}\right)
\end{aligned}
$$

where $M_{e}$ is the local Mach number at the edge of the boundary layer. Notice in Equation (39) that $C_{c p}$ has a constant value of 1.216 for local Mach numbers less than 1.52. The adjustment for higher Mach numbers is only a rough, first-order approximation and was determined from computations of turbulent boundary layers on a flat plate with freestream Mach number 2.4. Comparisons between experimental [17] and computed velocity profiles in the wake region of the boundary layer indicated that a value of 1.920 was significantly better, at free-stream Mach 2.4, than the 1.216 recommended for subsonic (incompressible) boundary layers [6]. The comparison is shown in Figure 2 where the distance from the wall $y$ is nondimensionalized by the momentum thickness $\theta$. The general effect of increasing $C_{c p}$ at the higher Mach numbers is to increase the turbulent eddy viscosity in the outer region, resulting in a thicker boundary layer and larger skin friction.

Normally 2000 to 3000 time-step iterations, involving 3 to 5 orders of magnitude reduction in the density residuals, are needed to achieve convergence to a steady-state viscous solution. The execution time of the DVC2D code on different computer systems was measured for a super- 


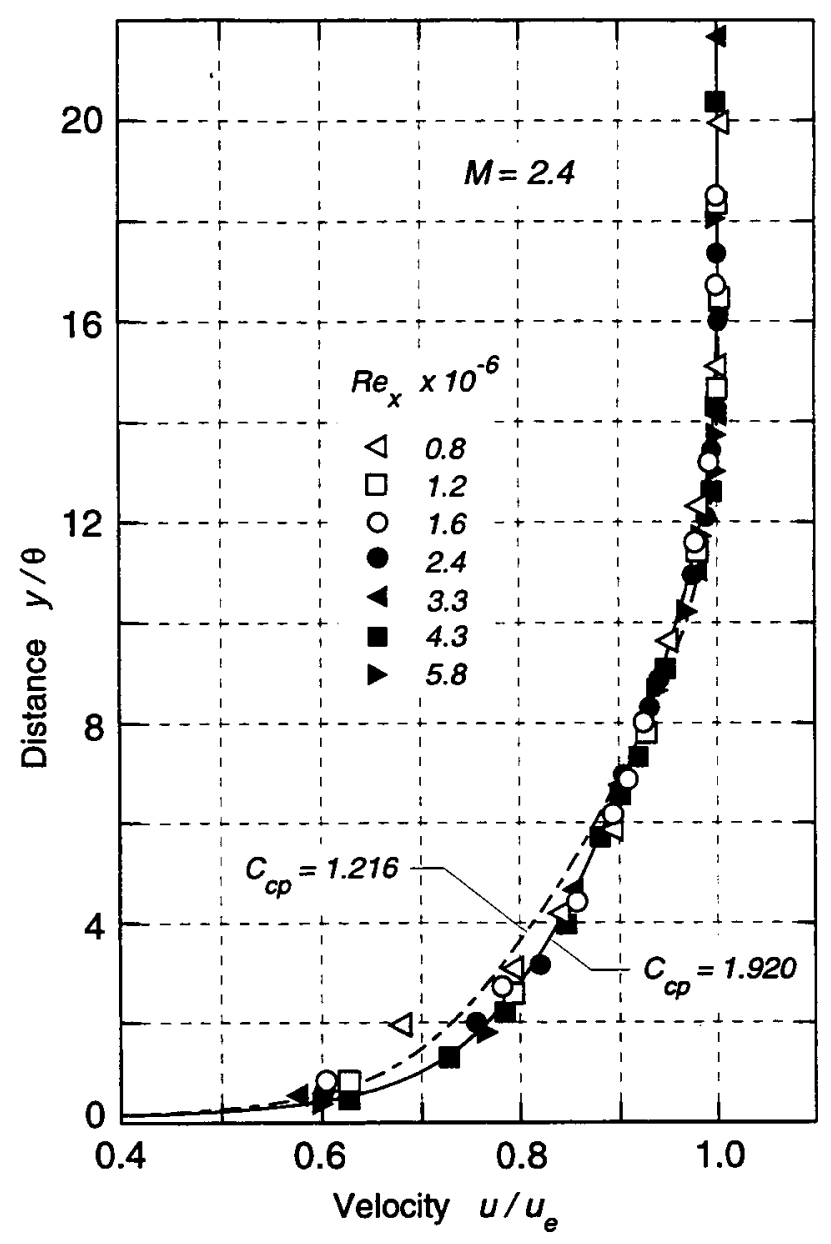

Figure 2 - Turbulent flat-plate boundary-layer velocity profile for freestream Mach 2.4 (experimental data reproduced from Reference 17)

sonic viscous flow simulation on a $150 \times 75$ grid. Based on 500 iterations, the execution times for different computers systems are listed in Table 1.

Core memory requirements for the code are small by current standards, being less than one megaword of random access memory (RAM) for a typical viscous simulation.

\section{Results}

\section{Waisted Body-of-Revolution}

The first set of results to be presented involves calculations of supersonic flow over a waisted body-ofrevolution. The experiment is described in detail in Reference 18 , so only a brief discussion introducing the basic features of the experiment will be provided here. The waisted body-of-revolution is shown in Figure 3, and as described in the reference was designed to provide certain desired variations in the streamtube area and streamwise
Table 1 - Approximate code execution times for various computer systems

\begin{tabular}{lc}
\hline $\begin{array}{c}\text { Computer system } \\
\text { (CPU) }\end{array}$ & $\begin{array}{c}\text { time/node/iteration } \\
(\mu \mathrm{s})\end{array}$ \\
\hline CRAY C-90 & 3.6 \\
CRAY Y-MP & 7.5 \\
SGI Power Challenge & 10.6 \\
(R8000 - 75MHz IP21) & \\
SGl 4D/440 [4 CPU's] & 61.4 \\
(R3000 - 40MHz IP7) & \\
SGI Indigo2 & 83.1 \\
(R4000 - 100MHz IP22) & \\
SGI 4D/35 & 176. \\
(R3000-36MHz IP12) &
\end{tabular}

a Silicon Graphics, Inc. Compiler options were selected for high levels of optimization and varied with the workstation model.

pressure-gradient, for the purpose of studying their influence on turbulent boundary-layer character and development.

Nominal test conditions at zero angle-of-attack included five different supersonic upstream Mach numbers ranging between Mach 1.4 and 2.8 , as well as two subsonic Mach number conditions, Mach 0.6 and 0.8 . The Reynolds number was varied for some tests, but measurements at the nominal freestream Reynolds number $R e_{L}$ of $1.0 \times 10^{7}$ based on the body length of $1.524 \mathrm{~m}(5 \mathrm{ft}$.) were made at each Mach number condition. Measurements of wall static pressure and local skin-friction were obtained along the length of the body, and boundary layer profiles were measured at a few selected axial locations.

Only two of the test conditions were examined computationally for the present work; namely, nominal upstream Mach numbers 1.4 and 2.8, with freestream Reynolds number $R e_{L}$ equal to $1.0 \times 10^{7}$. The same computational grid was used for simulations at both conditions, and it is shown in Figure 4. The grid dimensions are $150 \times 75$, but in the figure only every other $\zeta$-line (vertical line) is shown. The grid spacing normal and adjacent to

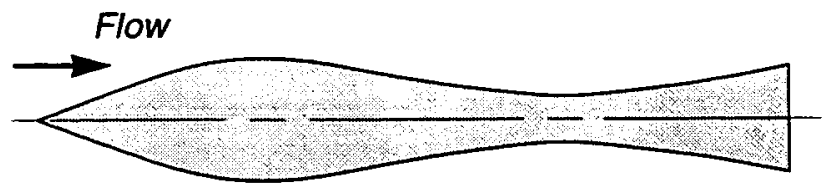

Figure 3 - Waisted body-of-revolution 


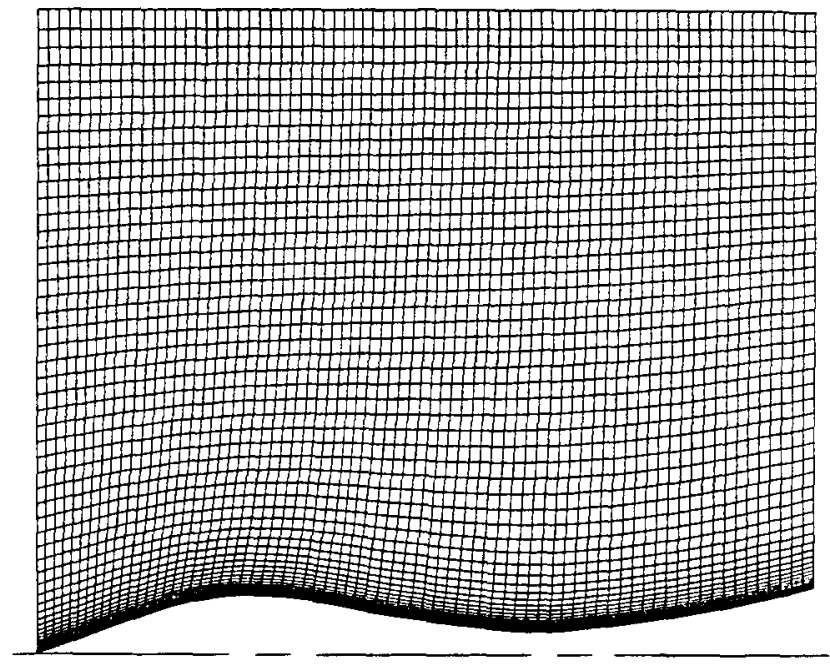

Figure 4 - Computational grid for waisted body-of-revolution (only every other vertical grid line shown)

\section{Contour increment 0.05}

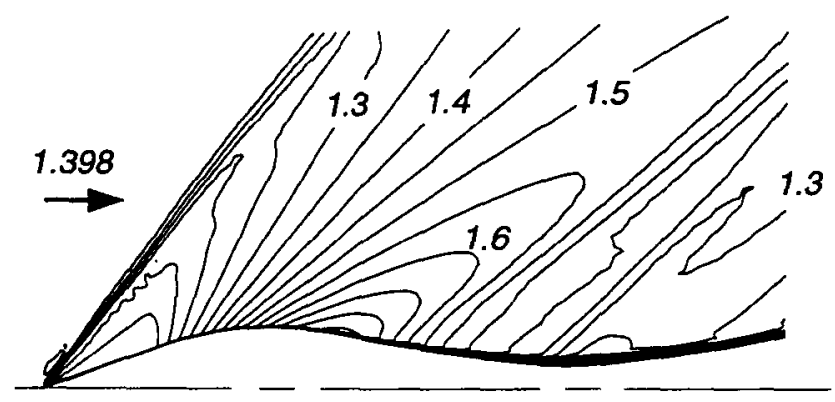

Figure 5 - Mach contours for computed flow field with upstream Mach 1.398

ized by body length, which is needed to imbed 3 to 4 interior points within the viscous sublayer.

The numerical simulation for upstream Mach number 1.4 was run assuming a turbulent boundary layer along the entire body, which is consistent with the experimental intent. As reported in Reference 18, a boundary layer transition trip of size $0.13 \mathrm{~mm}$ was attached $38.1 \mathrm{~mm}$ from the nose of the body. This was adequate for the Mach 1.4 condition, but the trip proved to be too small for the higher Mach number conditions as discussed in the reference, and later here with respect to the Mach 2.8 results.

Mach contours for the computed nominal Mach 1.4 case are shown in Figure 5, where it is indicated that the upstream Mach number is, more precisely, 1.398. Only about half of the computational domain is shown since the outer region is not important for the comparisons to be made. The outer (far-field) boundary was treated as an inviscid reflecting surface, but its distance from the body

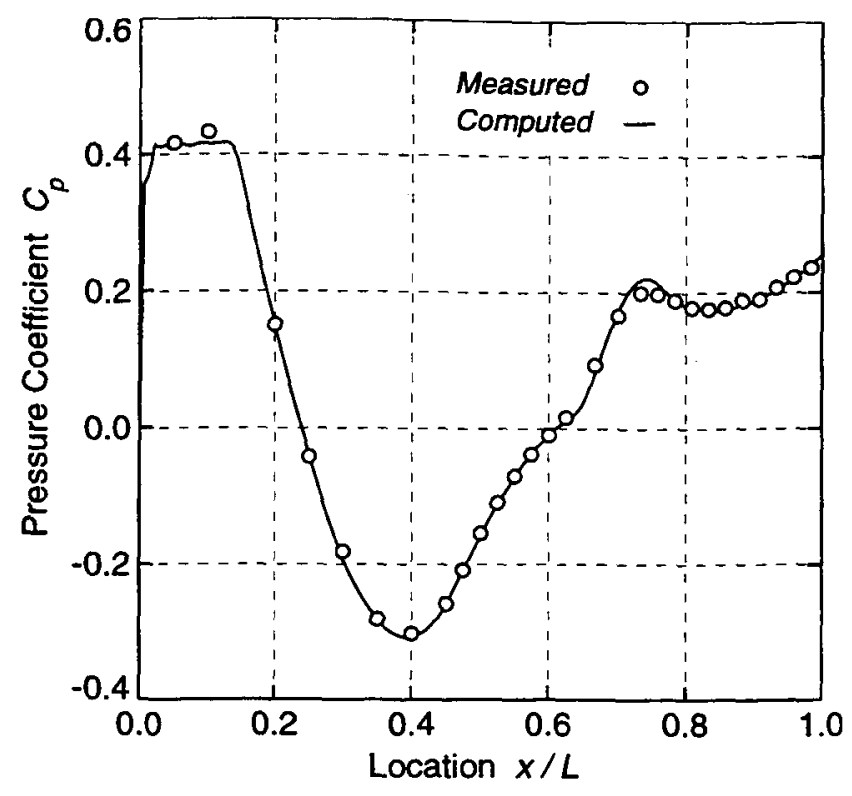

Figure 6 - Measure and computed pressure coefficient distributions for upstream Mach 1.398

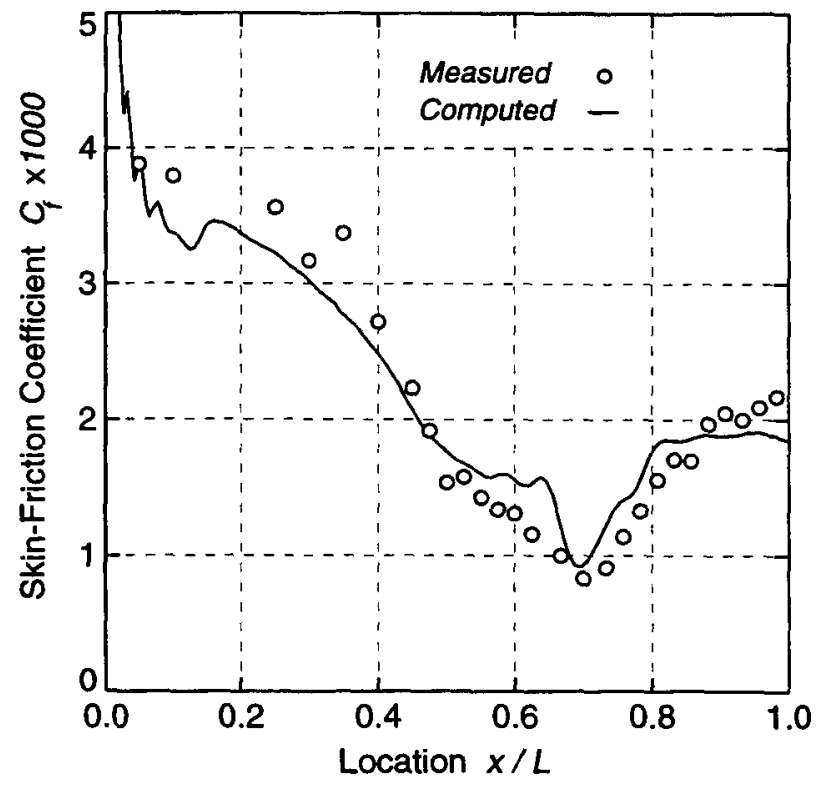

Figure 7 - Measure and computed skin-friction coefficient distributions for upstream Mach 1.398

is far enough away to avoid interference from the reflected waves.

The measured and computed distributions of pressure coefficient $C_{p}$ and of skin-friction coefficient $C_{f}$ are compared in Figures 6 and 7, respectively. As can be seen the agreement is good, especially for pressure coefficient. Note that the skin-friction coefficient is based on local 
dynamic pressure, rather than upstream dynamic pressure as might be expected [18].

Measured and computed boundary-layer profiles in terms of Pitot pressure are compared in Figure 8. In Reference 18 the profile data are given in terms of velocity ratios and Mach numbers, but to eliminate the added uncertainties inherent in these quantities due to data reduction assumptions, the measured Pitot pressures were recovered by reverse-calculation from the reported data. The agreement is generally good, although in the adverse pressure-gradient region, at $x / L=0.550$ and 0.700 , significant differences exist, as might be expected considering the well-known limitations of the turbulence model.

Mach number contours for the computed nominal Mach 2.8 case are shown in Figure 9, where as indicated the upstream Mach number is actually 2.799. Again, only about half of the computational domain is shown since the outer region is not important for the comparisons. The computed pressure coefficient distribution is compared with the measured distribution in Figure 10, and can be seen to agree well with it.

The measured and computed Pitot pressure profiles for the boundary layer at several axial locations are shown in Figure 11. In this case two computed solutions are shown; the dashed lines corresponding to an all-turbulent boundary layer, and the solid lines corresponding to a laminar/turbulent boundary layer (and to the computed results in Figures 9 and 10) with transition occurring at the onset of the adverse pressure gradient, at about $x / L=0.42$. As can be seen, the computer simulation with boundarylayer transition is in much better agreement with the data, confirming that the transition trip was too small at this Mach and Reynolds number. As discussed in Reference

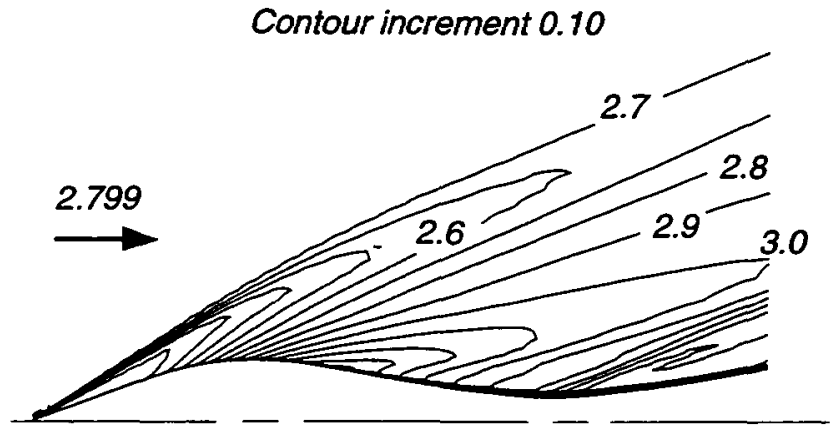

Figure 9- Mach contours for computed flow field with upstream Mach 2.799

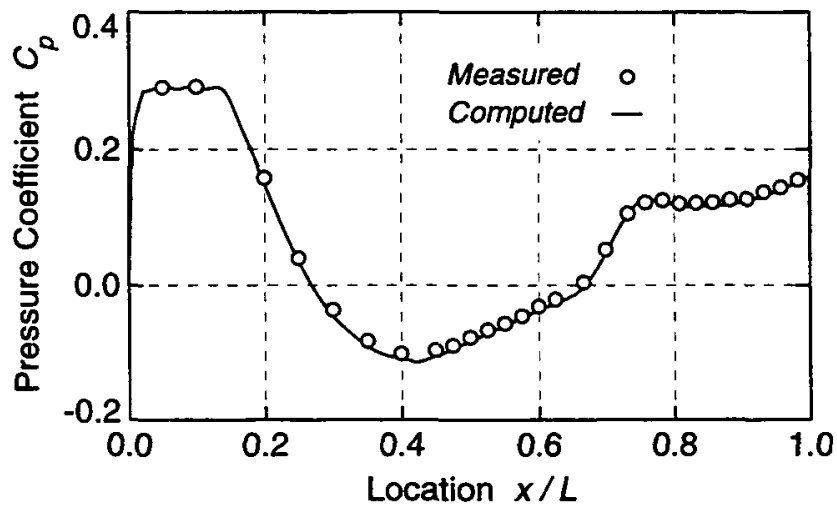

Figure $10-$ Measure and computed pressure coefficient distributions for upstream Mach 2.799

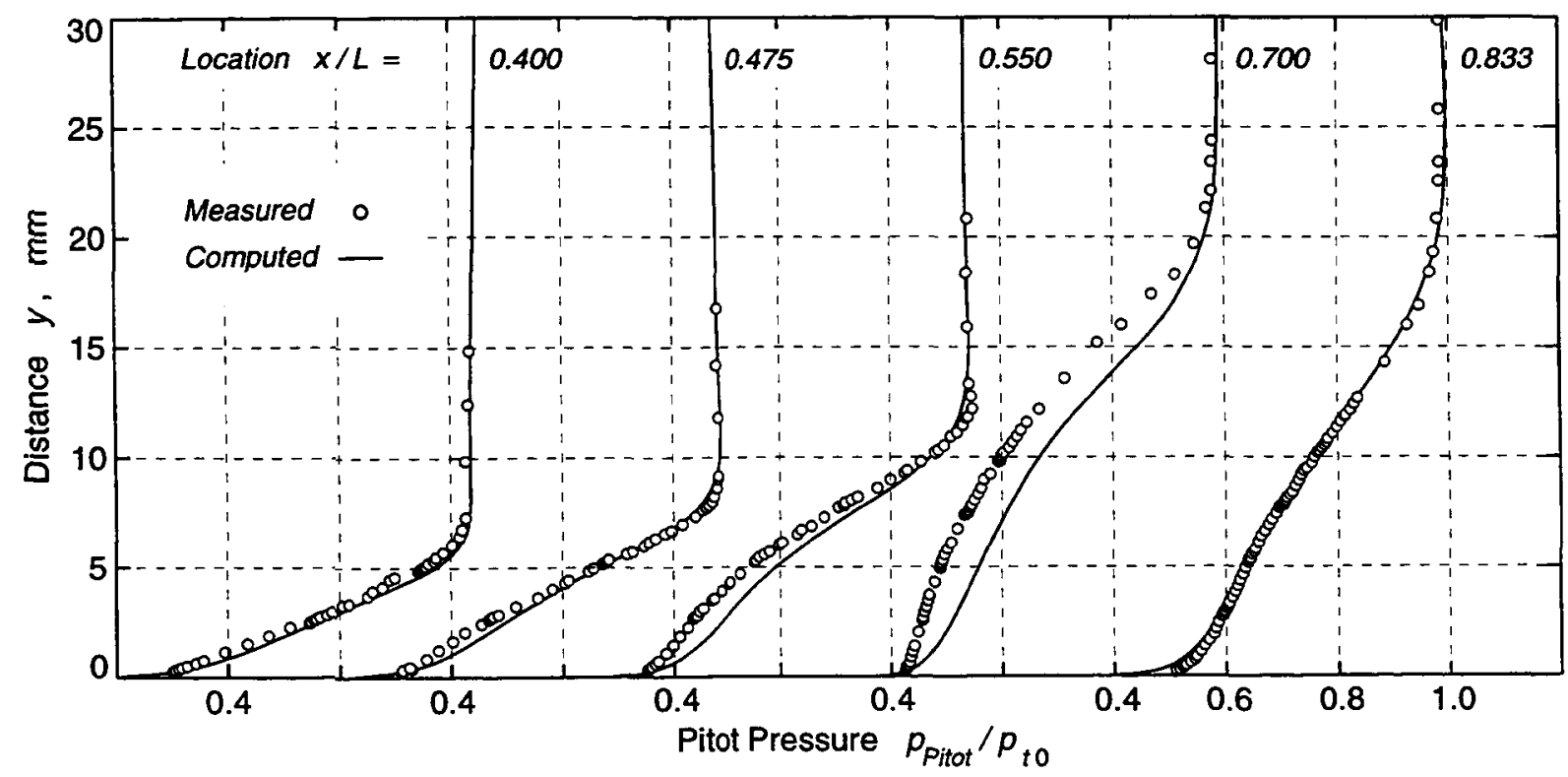

Figure 8- Measured and computed Pitot pressure profiles at several axial locations, for upstream Mach 1.398 


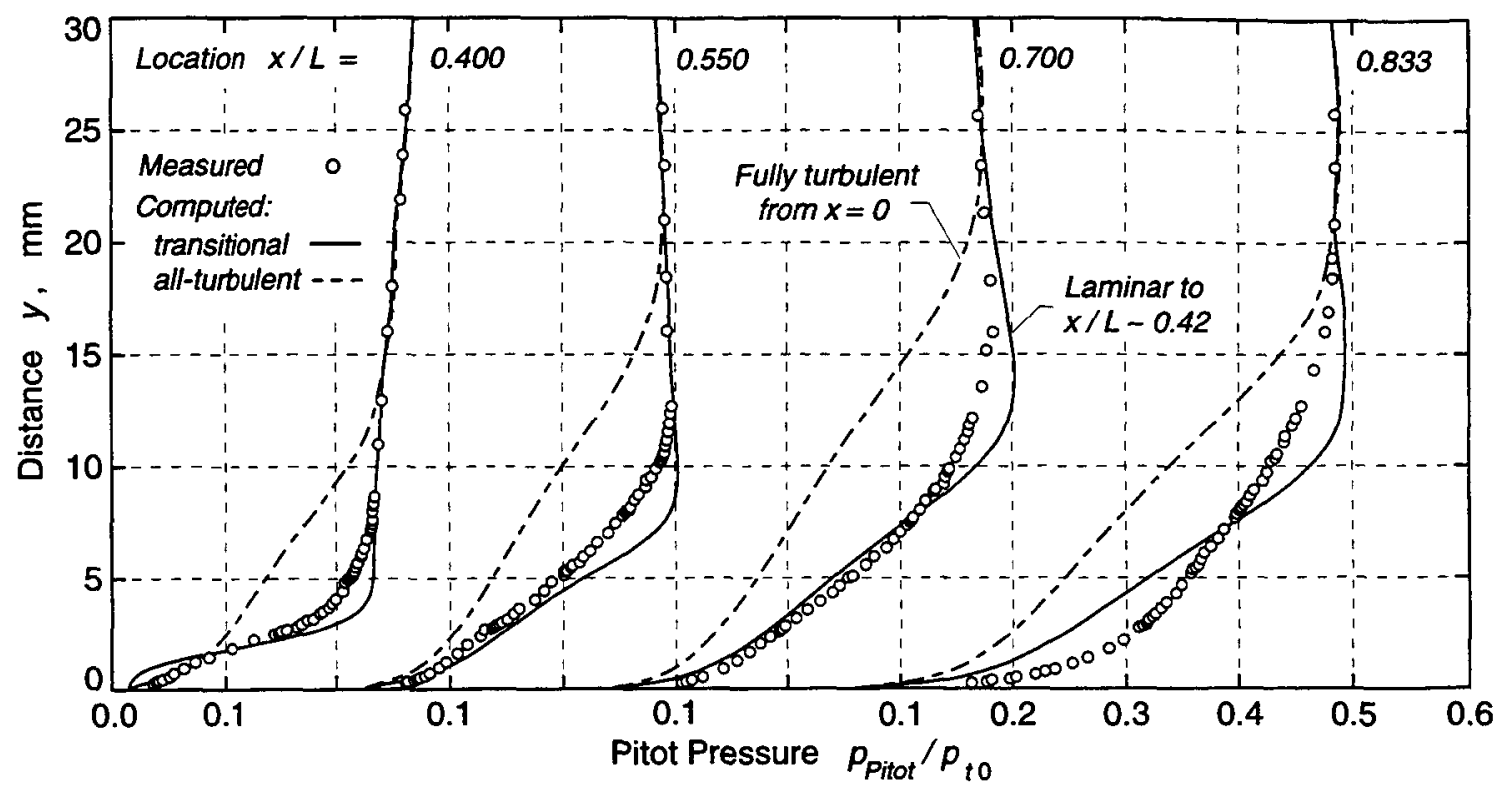

Figure 11 - Measured and computed Pitot pressure profiles at several axial locations, for upstream Mach 2.799

18 , this was established after the initial experiments, and a trip size on the order of $0.33 \mathrm{~mm}$ would have been needed to force transition at the nose of the body. Furthermore, because the boundary layer was not fully turbulent the skin-friction data are of questionable accuracy, at least over the front half of the body - the measurement technique was calibrated for only turbulent flow. For this reason, skin-friction results were not compared in this case.

Closer examination and comparison of the measured and computed profiles in Figure 11, particularly the profiles at $x / L=0.400$, reveals that the front half of the boundary layer probably was not completely laminar in the experiment. Some indications of turbulence are apparent in the shape of the experimental profile, but the turbulence may have been from the free-stream, rather than that arising from a transitional layer. In fact, a measurable influence of free-stream turbulence would be expected for a laminar boundary-layer in such a strong favorable pressure gradient. A more definitive evaluation cannot be given, however, since free-stream turbulence levels for the wind tunnel were not reported with the experimental results.

Finally, some information pertaining to both the Mach 1.4 and 2.8 numerical simulations might be included. First, as shown in Figure 12 the solutions behaved well numerically, with a fairly continuous decrease in the density-residuals by over four orders of magnitude in 2000 iterations. Both cases were run with a CFL number of 7.0, with residual smoothing coefficients at minimum values, and with low artificial viscosity levels - the first- and third-order artificial dissipation coefficients $\mu_{2}$ (scaled by $1 / 4$ ) and $\mu_{4}$ (scaled by $1 / 16$ ) were equal to 0.50 and
0.20 , respectively, for the Mach 1.4 flow, and 0.50 and 0.30 , respectively, for the Mach 2.8 flow. The fully turbulent cases were simulated by setting the turbulence model transition parameter $C_{\text {mutm }}$ [5] equal to zero, whereas the Mach 2.8 flow with transition was simulated by setting $C_{\text {mutm }}$ equal to 20 - the normal (default) value of 14 produced a "transitional" layer with the turbulence model

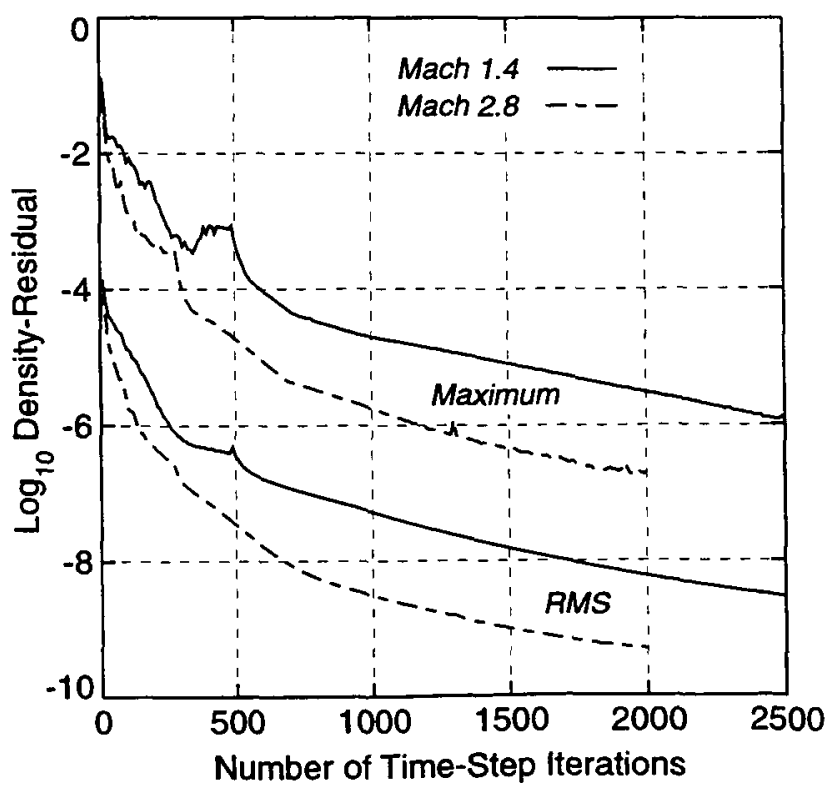

Figure 12 - Convergence histories for waisted body-ofrevolution flow field simulations 
turning briefly on, then off through the favorable pressuregradient region, and then on again as the adverse pressuregradient was encountered.

\section{Straight Circular Duct}

The next set of results to be presented involves the calculation of transonic flow through a straight circular duct of constant cross-sectional area. The experiment is described in detail in Reference 19 and was intended for studying the flow field of a normal-shock-wave/turbulentboundary-layer interaction. It was performed in a test section consisting of a tube with inside-diameter $D$ of 0.2477 $\mathrm{m}$, and length $L$ of $2.690 \mathrm{~m}$, and with the inside surface fabricated so as to be hydraulically smooth. Supersonic flow with a nominal Mach number of 1.5 entered the tube from a supersonic nozzle, and a normal shock wave was positioned within the tube at various locations. A hollow cylinder inserted into the exit of the tube was used to establish and position the shock wave. Because of wall boundary-layer growth a slight Mach-number gradient existed along the length of the test section, leading to some variation in the shock-incident Mach number as the shock position changed.

The test condition considered for the present work was one at which the most extensive measurement series was carried out [19-21]. It had a reference Mach number of 1.44 , at Reynolds number $R e_{D}$ of $5.43 \times 10^{6}$ based on the inside diameter of the tube - the reference point is at the boundary-layer edge just upstream of the shock wave. At this test condition both unsteady and mean-flow (slowresponse, steady-state) measurements were performed in the test section, although only the mean-flow data are used here. Mean-flow measurements included wall static pressures, and probe-surveys with Pitot pressure, static pressure, and total-temperature probes. Mean-flow shearstresses at the wall were determined from measurements with a Preston tube.

It is important to note that the survey and wall shearstress data were obtained by positioning the shock wave at various locations in the test section, relative to a single, fixed measurement location. The wall static-pressure distribution relative to the shock wave could be obtained in the same way; that is, by using a single static-tap at the probe location. However, it could also be obtained for a fixed shock position, or even several fixed shock positions, by using the array of static pressure taps distributed along the length of the test section. The different possibilities this allows seem to account for variations in the wall static-pressure distributions presented in the literature [1921] over a period of several years, for the same experiment.

In view of the experimental approach, comparisons between measured and computed results are somewhat problematic. There is a significant quantitative difference between the (shock-relative) flow field for a fixed shock location, and that for a variable shock location. Nevertheless, comparisons are made, but with care in attempting to draw valid inferences.
The numerical simulation of the flow field was sensitive to small changes in flow conditions. This was expected for a transonic flow field with strong viscous/ inviscid interaction, where even small changes in upstream or downstream conditions would substantially alter the shock position. Consequently, it was important that the supersonic upstream boundary condition to the computational domain closely match that of the experiment. This could be done for only one of the experimental shock positions, which was the position where the shock wave was located farthest downstream.

A few assumptions and simplifications were involved in using the measured shock-incident flow profile to determine the upstream boundary condition for the computational domain. Briefly, the turbulent boundary layer profile was constructed using the measured skin-friction coefficient $C_{f \infty}$ of 0.0018 in conjunction with the measured velocity profile data [21]. Near the wall, for dimensionless wall distances $y^{+}$less than 50 , Spalding's innerlaw composite formula [22] was used to define the velocity profile. Between that region and the innermost measured velocity data points, a cubic-spline interpolation of dimensionless velocity $u^{+}$versus $\ln \left(y^{+}\right)$was used to fill in the rest of the profile. The static pressure was assumed constant at the reference value across the boundary layer, and the temperature profile was approximated by assuming an adiabatic wall and using the Crocco-Busemann [23] approximation:

$$
\frac{T}{T_{\infty}}=\frac{T_{a w}}{T_{\infty}}-r \frac{\gamma-1}{2} M_{\infty}^{2}\left(\frac{u}{u_{\infty}}\right)^{2}
$$

where $T_{a w}$ is the adiabatic wall temperature, and the turbulent recovery factor $r$ was given a value of 0.895 . The resulting density profile agrees well with the measured density profile, as shown in Figure 13, where the distance from the wall $y$ is nondimensionalized by the tube insidediameter $D$. (The resulting velocity profile will be shown later along with several downstream profiles.) The profile in the free-stream, between the boundary-layer edge and the centerline, was constructed using the measured velocity profile in conjunction with the measured plenum total temperature and the reference static pressure, assuming homentropic flow.

The computer simulation was performed on two different grids, both of dimensions $150 \times 50$, with the same grid spacing from wall-to-centerline. The grid spacing normal and adjacent to the wall is about $2.0 \times 10^{-5}$, as nondimensionalized by tube diameter, which imbeds at least 3 to 4 interior points within the viscous sublayer. The upstream halves of the two grids are shown in Figure 14 along with Mach contours for the corresponding computed solutions. The so-called "coarse grid" has uniform spacing in the axial direction, whereas the "fine grid" has been locally (and manually) refined around the normal shock 


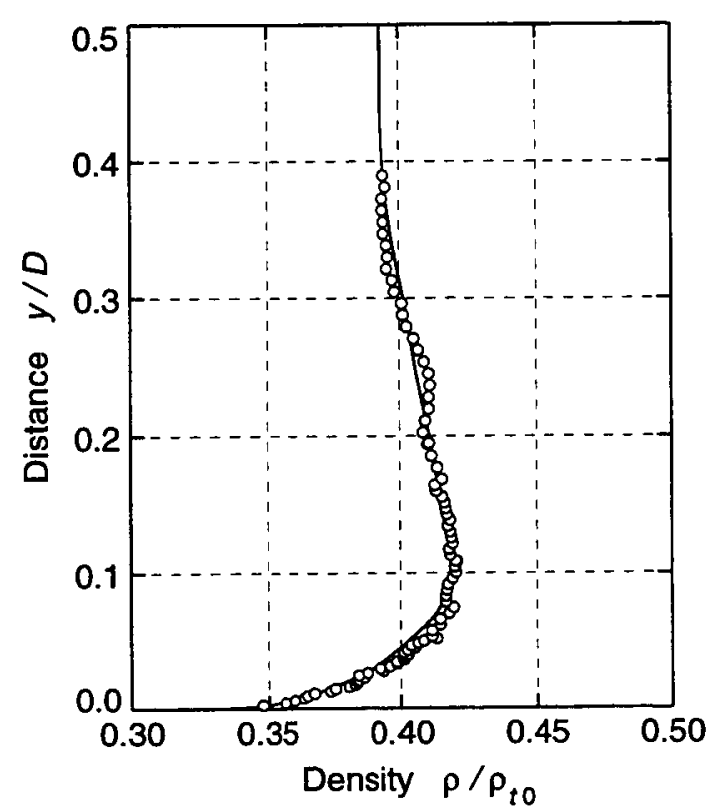

Figure 13- Measured and specified density profiles at inlet boundary with reference Mach 1.44
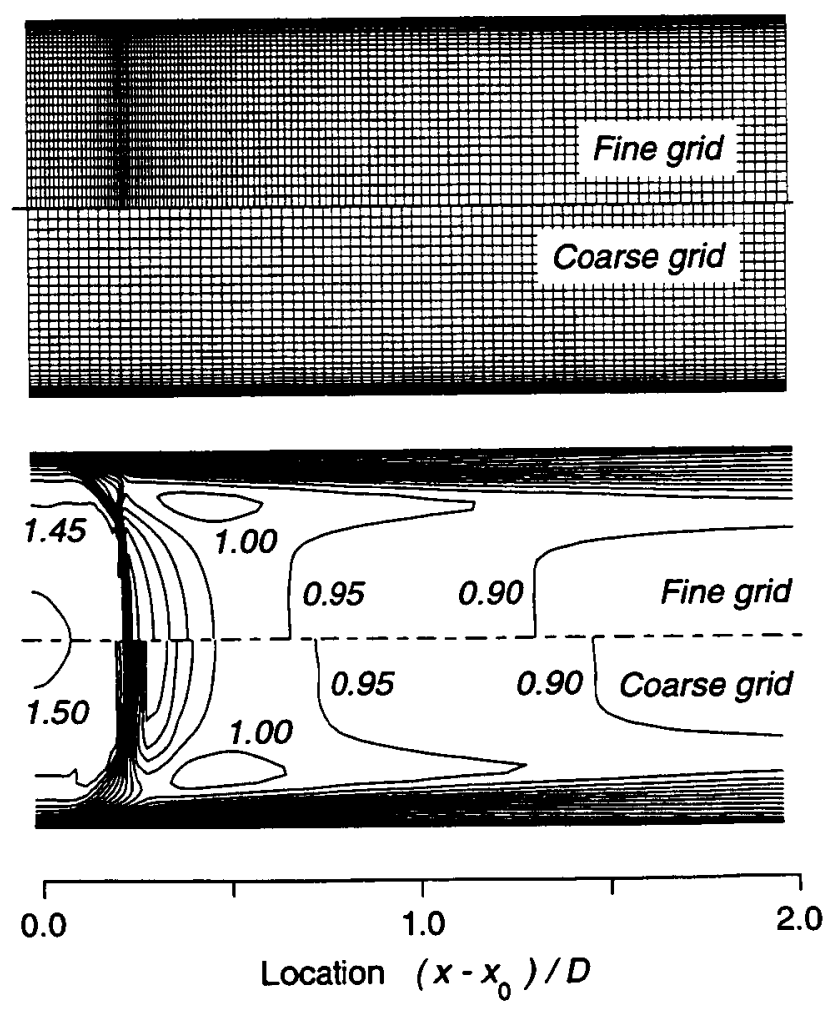

Figure $14-$ Computational grids and Mach number contours for computed flow fields

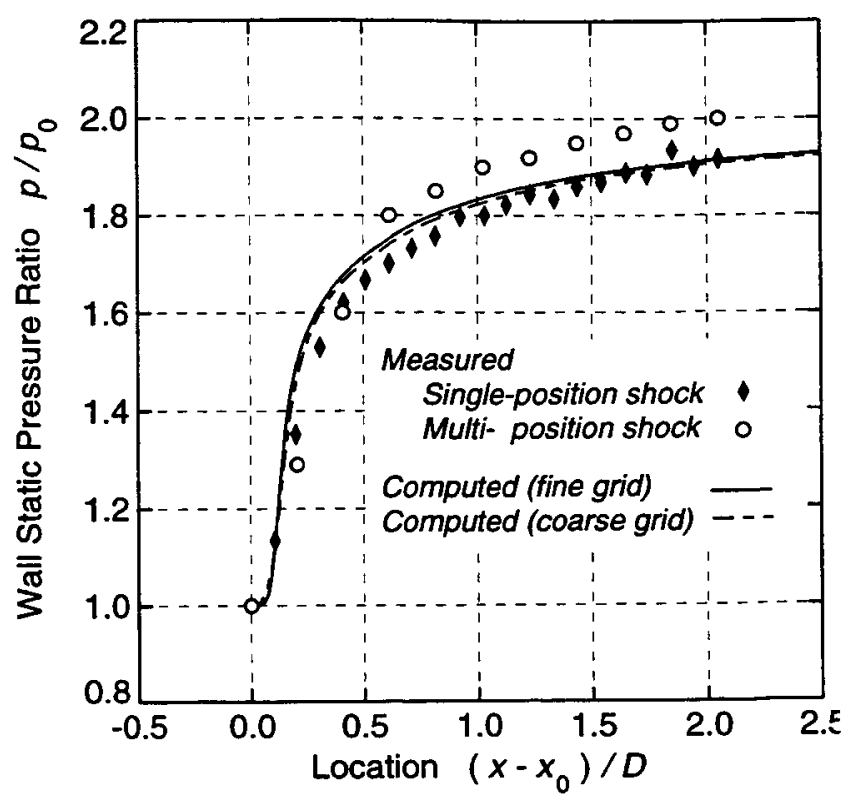

Figure 15 - Measured and computed pressure coefficient distributions for reference Mach 1.44

for better shock resolution. The coarse grid is considered more typical of what would be used in routine code application where the location of strong shock waves is often not fixed, nor known a priori.

To obtain the solutions in Figure 14 it was necessary to iteratively vary the exit static pressure until the shock was located just downstream of the inlet boundary. However, the shock was not pushed so far upstream that the beginning of the shock/boundary-layer interaction region (at $x=x_{0}$ ) reached the inlet boundary. In both cases the shocks are close enough to the inlet boundary that the flow profile does not change significantly between inlet and shock, as can be inferred from the Mach contours.

The simulations were run with a CFL number of 7.0, with residual smoothing coefficients at minimum values, and with (scaled) first- and third-order artificial viscosity coefficients of 0.50 and 0.25 , respectively. Due to the strong viscous/inviscid interaction and marginal steadystate flow-field stability, around 20,000 iterations were required to achieve full convergence (5 to 6 orders of magnitude reduction in the density-residuals) at a particular exit pressure. Such a large number of iterations is very abnormal and should be considered peculiar to this type of problem.

Comparisons between the measured and computed wall static-pressure distributions are shown in Figure 15. The two sets of experimental wall pressure data correspond to two different measurement approaches; one with the shock wave at a single location [20] in the test section (diamonds), and the other with the shock wave relocated to various positions [21] relative to the probe-survey location (circles). The case with a single shock position is, in 
principle, most like the computation, but for that case the exact inlet flow profile is not known (and therefore not used for the computation). In general, the computed distributions for the coarse and fine grid are almost identical to each other, and are in basic agreement with the experimental data.

The measured and computed skin-friction coefficient distributions are compared in Figure 16. As with the wall static-pressure results the two computations are almost

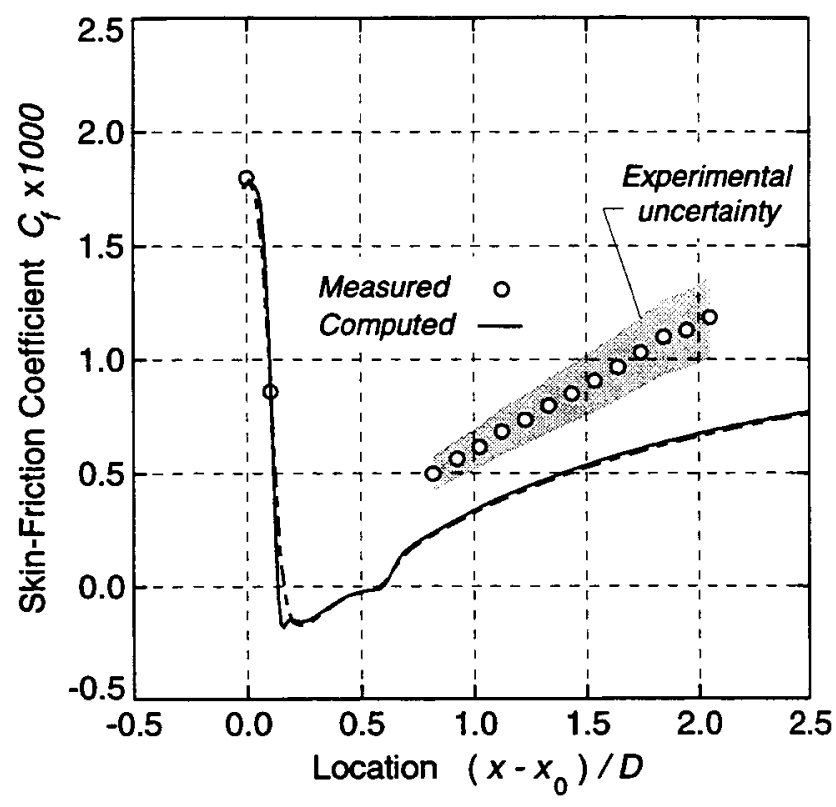

Figure 16 - Measured and computed skin-friction coefficient distributions for reference Mach 1.44 identical to each other, but here their agreement with experiment is not as good. The agreement might, however, be considered fair from the standpoint of accuracy requirements for much engineering work, and in view of the strong adverse pressure-gradient. Results very similar to this were reported by Viegas and Horstman [20], and the failure of the computation to more accurately simulate the flow field lies with the turbulence model. Reasons for the lack of accuracy are well-known and understood in the field of turbulence-modeling research and development; for example, see Reference 24.

Notice that the computed solutions indicate a relatively small, weak separation "beneath" the shock wave, that is, between $\left(x-x_{0}\right) / D$ equal to 0.2 and 0.6 , with a rapid increase in skin friction once reattachment occurs. The experimental results seem inconclusive in this regard, with the original study indicating that there was separation [19], but later work suggesting that separation probably did not occur [20].

Velocity profiles at several axial locations are compared in Figure 17. Also shown in the figure is the upstream profile, at $x=x_{0}$, which is a fit to the experimental data as explained earlier. Consistent with the static pressure and skin-friction results just presented, the agreement is generally fair between the measured and computed velocity profiles.

A basic summary of the results helps to clarify the comparison. First, the computed flow in the interaction region exhibits an initial compression (pressure rise) which is too rapid, and which is followed by too gradual of a subsonic flow diffusion (pressure rise). The differing rates of subsonic diffusion can be seen in the wall staticpressure gradients, as well as in the progression of velocity

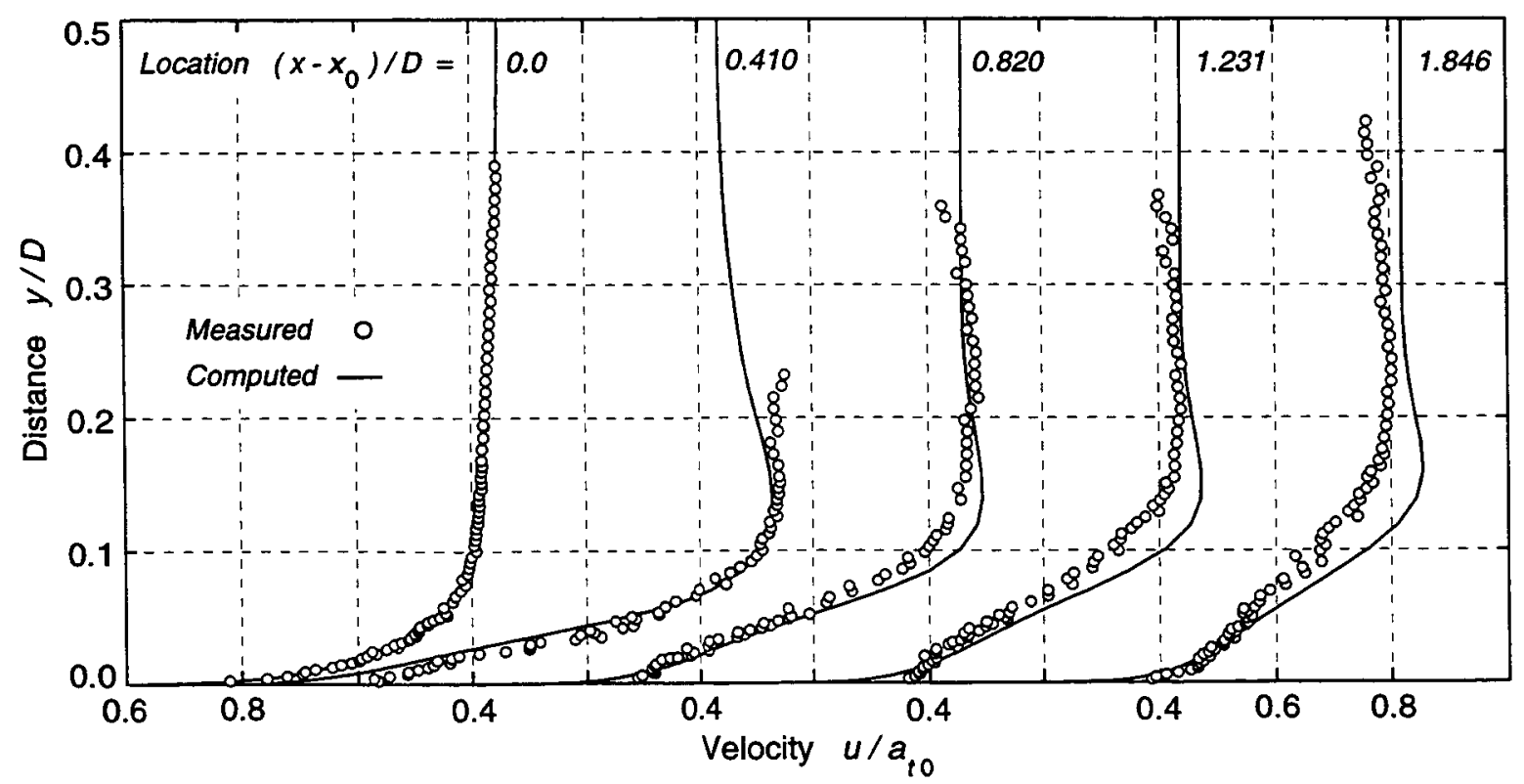

Figure 17 - Measured and computed velocity profiles at several axial locations, for reference Mach 1.44 
profiles (see Figure 17, where the experimental freestream velocity level decreases more rapidly with distance downstream than does the computed velocity level). The measured values of skin-friction are higher than those computed, consistent with and corresponding to a larger decrease in boundary-layer-wake momentum, i.e., lower velocities.

In Figure 17, one particular feature is worth further discussion, namely, the condition at the boundary-layer edge for the profile just downstream of the shock wave, at $\left(x-x_{0}\right) / D=0.41$. At this location the computed velocity (fine grid solution) at the boundary-layer edge is slightly supersonic at about Mach $1.02\left(u / a_{t}=0.96\right)$,

compared to a measured value of Mach 1.04. This computed (see Figure 14) and measured region of supersonic flow at the edge of the boundary layer just downstream of the normal shock, sometimes called a "supersonic tongue", is mentioned in Reference 19 , and has been observed by others for similar flows [25-27]. The supersonic tongue results from the bifurcated, or "lambda", shock structure at the base of the normal shock, and apparently exists only with shock-incident Mach numbers greater than about 1.4. Within the lambda shock system the compression occurs more gradually, as a supersonic diffusion, with less total-pressure loss.

\section{Straight Radial Duct}

The next simulation case to be presented involves swirling supersonic flow through a strong shock in a straight radial duct. The primary purpose of this test case is to demonstrate the application of the method to an axisymmetric flow field with swirl, while at the same time allowing an assessment of the solution accuracy under such conditions. Documented experimental data for tests involving compressible swirling axisymmetric flow fields are difficult to find, so an inviscid "two-dimensional" simulation has been run for which there is an exact analytical solution to compare with. A set of solutions involving a strong shock in the duct was selected mostly for the sake of added interest, but also to demonstrate the accurate shock-capturing capability in the radial direction.

The geometry and grid for the simulation can be simply described and so they need not be shown. Briefly, the duct is straight and radial, extending from a dimensionless radius of 1.0 to a radius of 2.0 , with an aspect ratio $(\Delta z) /(\Delta x)$ of 10 . The grid had dimensions $150 \times 15$, evenly spaced in both directions ( 15 nodes in the $\zeta$-direction, or $x$-direction, from wall-to-wall). The simulated flow field was two-dimensional with a zero velocity component in the $x$-direction; that is, the inflow was a swirling radial flow with no variation wall-to-wall.

Four different solutions, corresponding to three different subsonic exit static pressures and one all-supersonic case, were computed. The flow at the grid inlet was fixed at a radial Mach number component of 1.05 , with azimuthal (swirl) component 0.95 , giving a resultant inlet
Mach number of 1.416 at a direction 42.14 degrees from radial. The simulations were run for 2000 iterations with a CFL number of 7.0 , with (scaled) first- and third-order artificial viscosity coefficients of 1.1 (up to 1.5 ) and 0.50 , respectively. The residual smoothing coefficients in the $\xi$ direction were at minimum values, while some additional smoothing in the $\zeta$-direction was necessary to obtain good convergence behavior - a continuous decrease in the density-residuals by about five orders of magnitude.

The computed results are compared with the exact solutions in Figure 18, where graphs of Mach number, flow angle, and total pressure ratio are shown. Except in close proximity to the shocks, the agreement is virtually "exact", as might be expected. Even through the shock waves, however, the agreement is very good in terms of both shock location and shock strength. Oscillations upand down-stream of the shocks are minimal for a numerical scheme employing central-differences, although to avoid stronger oscillations it was necessary to increase the first-order artificial viscosity coefficient somewhat (from 1.1 to 1.5 ) as the shock strength increased.

\section{Annular-to-Circular Diffuser Duct}

The last simulation case to be presented involves swirling subsonic flow in an annular-to-circular diffuser duct. In this case no comparison is made with experiment or theory, the main purpose being to demonstrate the method for a swirling flow with viscous effects. Although it would be preferable to have a comparison with experimental data, as already mentioned it is difficult to find documented data for experiments involving compressible swirling axisymmetric flows.

The geometry with grid for the annular-to-circular duct is shown in Figure 19, where locations of three axial stations downstream of the centerbody are also indicated. The outer radius $z_{o}$ of the duct is constant, the inner-outer wall radius ratio at the duct inlet is 0.50 , and the centerbody is a cylindrical section of length $x / z_{o}$ equal to 0.50 followed by a tangent ogive with length-to-diameter aspect ratio 3.80. The aft end of the ogive is spherically rounded with an end-radius $r / z_{0}$ of 0.066 . The dimensions of the grid are $115 \times 65$, with a spacing normal and adjacent to the walls (and centerline) of about $5.0 \times 10^{-5}$, as non-dimensionalized by outer radius, imbedding 2 to 3 interior points within the viscous sublayer.

For the simulation, the flow at the duct inlet was a swirling free-vortex with a mid-height Mach number of 0.405 and a flow direction 28.7 degrees from axial. Turbulent boundary-layer profiles were specified for the inner and outer walls of the inlet, each layer with a thickness of 20 percent of annulus height. The downstream static pressure at the outer wall was 93.5 percent of the inlet total pressure, and based on the mid-height inlet Mach number and duct outer-diameter, the Reynolds number for the simulation was $4.44 \times 10^{6}$. 

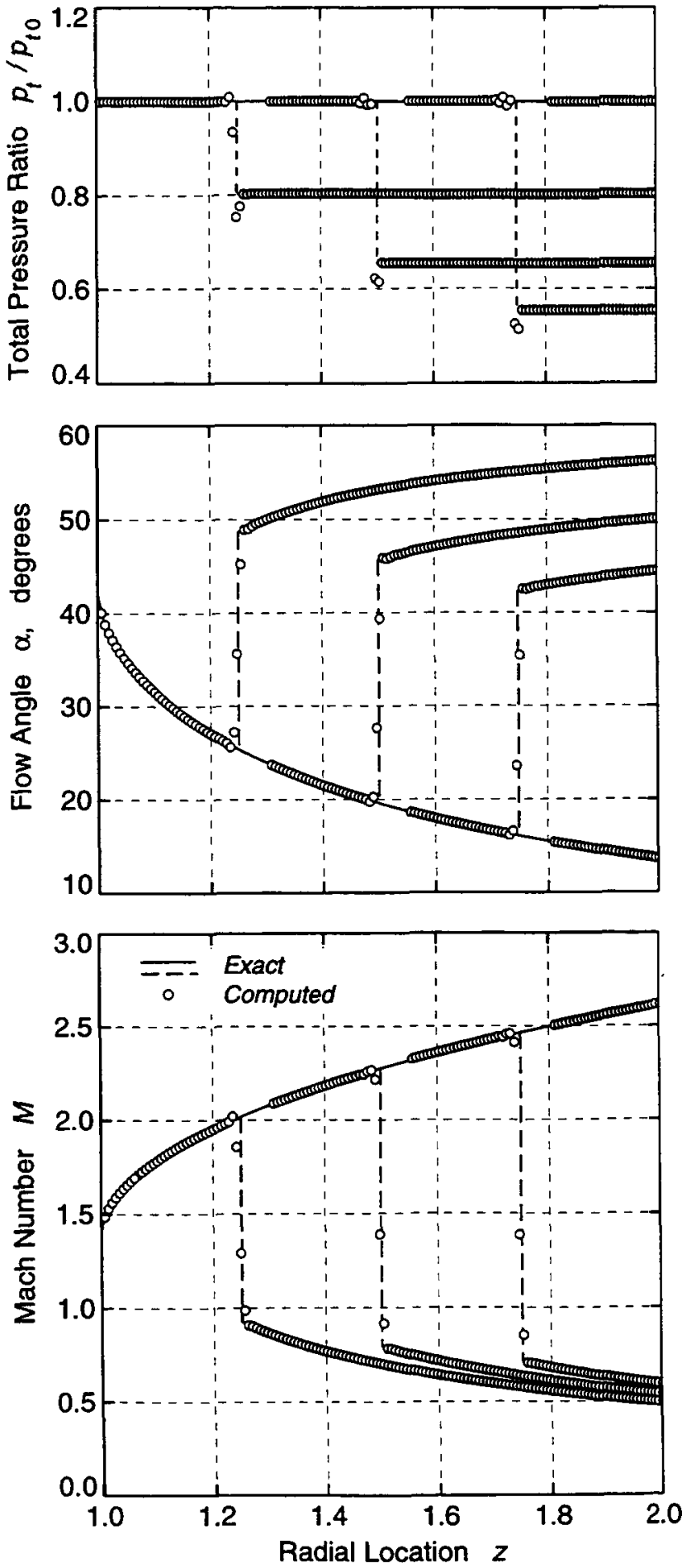

Figure 18- Exact and computed solutions for swirling inviscid flow through a strong shock wave in a straight radial duct

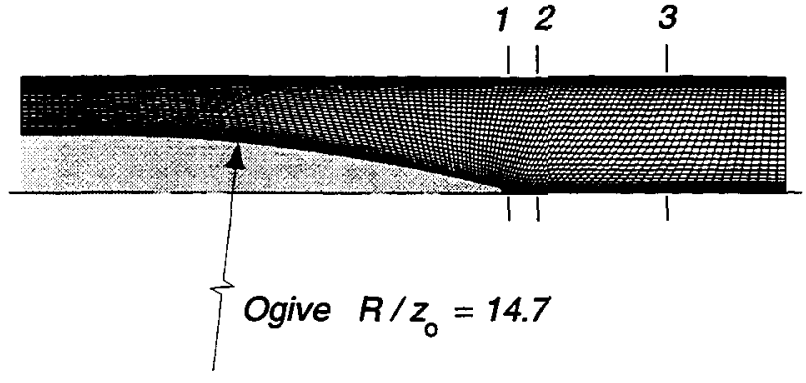

Figure $19-$ Geometry and computational gird for annular-to-circular diffuser duct

Contour increment 0.05

Mach Number $M$ 0.37

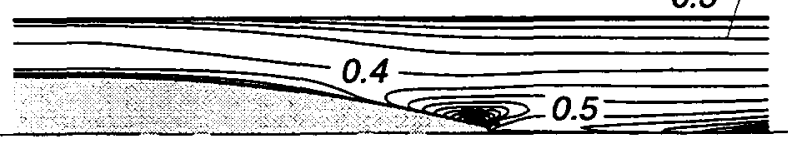

Axial Velocity $u / a_{t 0}$
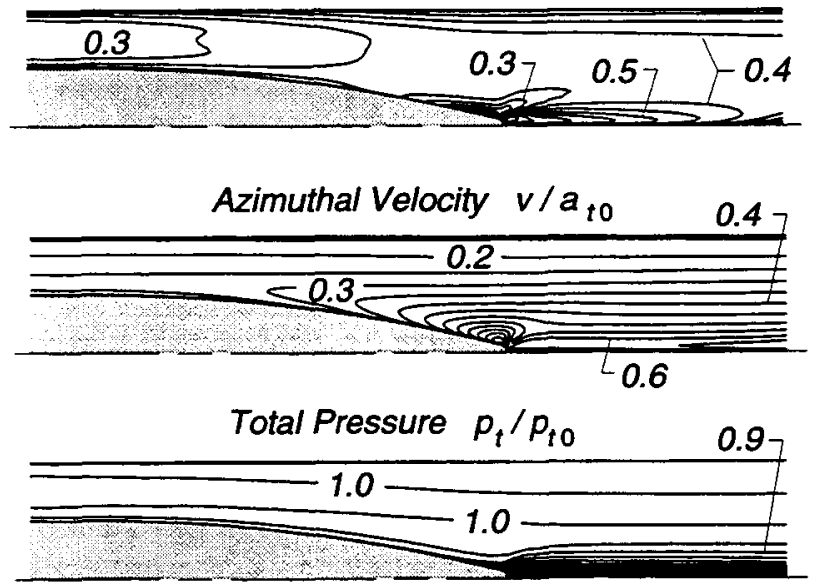

Figure 20-Contours of various flow quantities for the computed flow field

Contour plots for the simulated flow field are shown in Figure 20, and graphs of axial and azimuthal velocity versus radius are shown in Figures 21 and 22, respectively, for the three axial stations indicated in Figure 19. A discussion summarizing these results follows.

To begin, it might be pointed out that except for the wall boundary-layer regions and the region behind the base of the centerbody, the flow field remains closely irrotational (homentropic free-vortex). Some diffusion occurs in the outer half of the duct, as expected due to the area increase, whereas in the inner half there is no diffusion. The lack of a diffusion along the duct inner half is due to the decreasing radius which causes the azimuthal velocity 


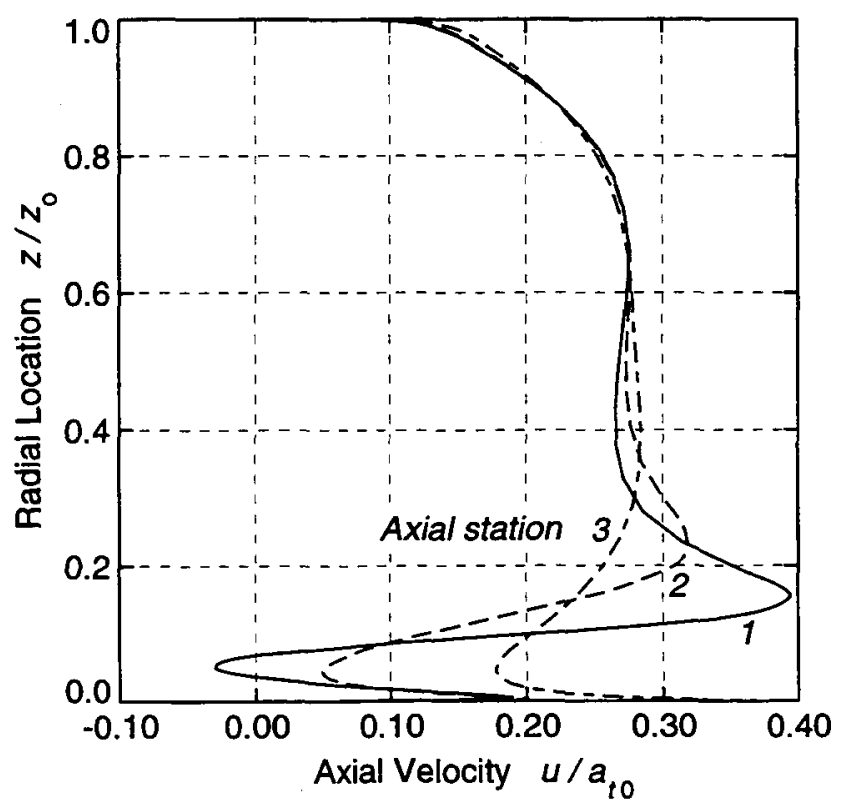

Figure 21 - Radial distributions of axial velocity at axial stations downstream of centerbody

to increase considerably, azimuthal velocity being inversely-proportional to radius. That is, the flow progressing downstream toward the base of the centerbody remains part of a free-vortex field - notice the horizontal orientation of the azimuthal-velocity contour lines, in conjunction with an almost uniform total-pressure field except within the wall boundary layer.

Far more intriguing than the above, however, is the flow at and behind the base of the centerbody. At the aft end of the centerbody an abrupt change in the flow can be seen to occur, downstream of which a large total-pressure deficit exists, especially near the centerline. This transition is ostensibly a form of vortex breakdown, the occurrence of which is to be expected under the flow conditions simulated. In discussing various types of vortex-breakdown transitions, Keller [28] has written the following with respect to this particular type: "It can be observed in isolation by directly generating a supercritical hollow-core vortex in an annular vortex tube with a suddenly ending center body." Vortex break-down phenomena and theory are well beyond the scope and intent of this work, but a few descriptive comments related to the simulated flow solution might be of general interest.

References dealing with the subject of vortex breakdown [28-31] describe it as a wave-like phenomenon, analogous to abrupt-transition phenomena in compressible gas flows and in open-channel (gravity, free-surface) flows. In the numerical test case under consideration, the vortex-breakdown transition occurs between the so-called supercritical vortex flow state upstream of the centerbody base, and a subcritical vortex flow state downstream of the centerbody. Thus, it is analogous to a strong shock wave

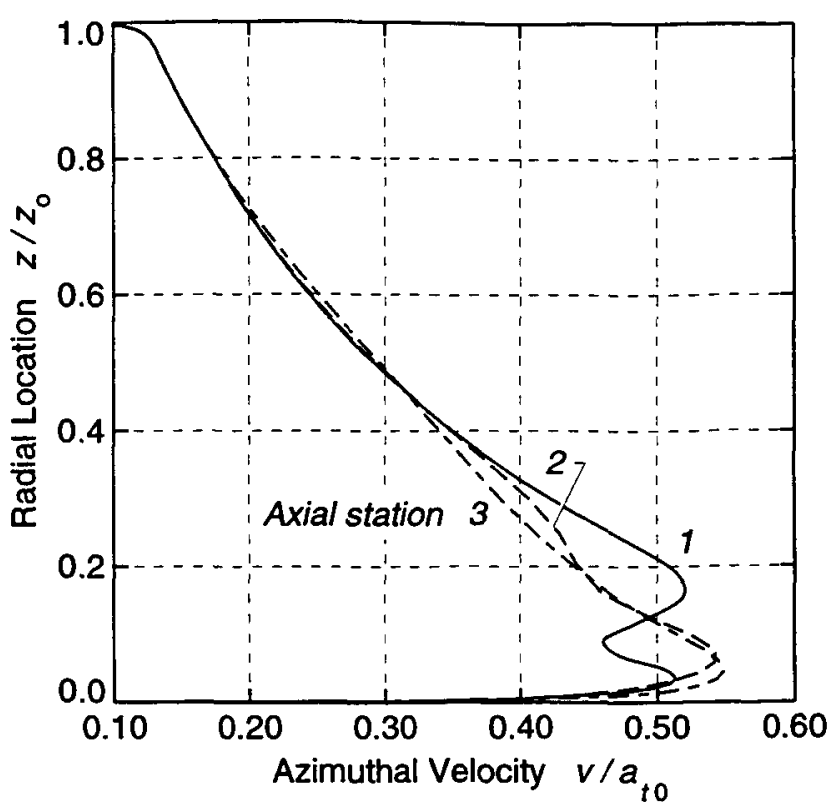

Figure 22 - Radial distributions of azimuthal velocity at axial stations downstream of centerbody

in a compressible gas flow, or to a hydraulic jump in a shallow open-channel water flow. The result of the transition is, locally, a substantial decrease in Mach number, azimuthal velocity, and total pressure (see Figure 20). Although not shown, a sudden jump to large amounts of vorticity also accompanies the transition, with the azimuthal velocity distribution near the centerline approaching that of a solid-body rotation. The profiles in Figure 22 help to show this, as well as the reduction in azimuthal velocity levels below what would exist for an irrotational flow field.

The axial velocity field downstream of the vortexbreakdown transition is difficult to discern from the above figures only, so in Figure 23 a velocity-vector plot is also provided. As can be seen in Figure 23, or even partially in Figure 21, the streamwise flow stagnates in a "bubble" just behind the transition. The velocities within the bubble are

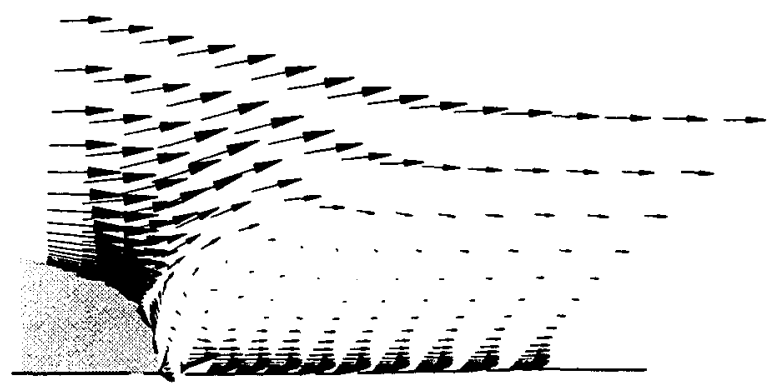

Figure 23 - Velocity vector field in the vortex-breakdown region 
still relatively large, however, but only in the azimuthal direction (see Figure 22, station 1). In effect, a portion of the flow just swirls around the centerline without going anywhere, while producing a large area-blockage to the axial flow.

To conclude this last case, a few comments about the numerical simulation can be added. The simulation was run with a CFL number of 5.0, with residual smoothing coefficients somewhat above minimum values. The (scaled) first- and third-order artificial viscosity coefficients had a value of 0.50 . Relatively low meridional Mach number levels for the flow field (weak compressibility) caused slower-than-normal numerical convergence, especially near and on the centerline where the flow field developed somewhat slower. The solution achieved full convergence ( 4 to 5 orders of magnitude reduction in the density-residuals) at around 4,500 iterations. The simulation was somewhat temperamental regarding the selection of numerical parameters, apparently due to the "viscous" grid spacing at the centerline, and perhaps aggravated slightly by the unusually high vorticity concentrated near there. As a result, some additional residual smoothing was necessary for stability. An additional point might be mentioned regarding this; namely, that stability problems were encountered in some subsonic simulations where initial azimuthal velocities were not near quasi-steady equilibrium values. Under such conditions, even when a converged solution was achieved the number of iterations was abnormally large. A convenient way to systematically circumvent this problem was found, and involves initially setting the azimuthal velocities everywhere to zero except at the upstream boundary. As the flow field subsequently develops the circumferential momentum convects downstream without causing numerical problems.

\section{Concluding Remarks}

A two-dimensional Navier-Stokes code has been developed for rapid numerical simulation of axisymmetric flow fields, including flow fields with an azimuthal velocity component. The azimuthal-invariant Navier-Stokes equations in a cylindrical coordinate system are mapped to a general body-fitted coordinate system, with the streamwise viscous terms then neglected by applying the thinlayer approximation. Turbulence effects are modeled using an algebraic model, typically the Baldwin-Lomax turbulence model, although a modified Cebeci-Smith model can also be used. The equations are discretized using central finite differences and solved using a multistage Runge-Kutta algorithm with a spatially-varying time step and implicit residual smoothing.

Results were presented for calculations of supersonic flow over a waisted body-of-revolution, transonic flow through a normal shock wave in a straight circular duct of constant cross-sectional area, swirling supersonic (inviscid) flow through a strong shock in a straight radial duct, and swirling subsonic flow in an annular-to-circular diffuser duct. Comparisons between computed and experimental results were in fair to good agreement, demonstrating that the viscous code can be a useful tool for practical engineering design and analysis work.

The code might also find useful application as a platform for turbulence model development and assessment. Along this line, some near-term future development of the code is planned. Specifically, recent efforts by Chima [32] in implementing the $\mathbf{k}-\omega$ two-equation turbulence model [33] will be incorporated into the DVC2D code. The increase in execution time for the code is estimated to be around 15 percent, and core-memory requirements will be extended by about 30 percent.

As a further enhancement, some investigation is currently underway to examine the feasibility of incorporating a preconditioning scheme into the solver [34-36]. This feature would improve the code considerably by extending its applicability to flow fields with very weak compressibility. A current limitation of the code, common to many codes of this type, is that convergence problems become acute when relatively large regions in the computational domain are nearly incompressible. The presence of high azimuthal velocities has been observed to provide no relief in this regard; that is, compressibility as introduced solely through circumferential momentum is apparently insignificant with respect to the influence of compressibility on numerical convergence.

\section{References}

1. Nietubicz, C. J., Pulliam, T. H., and Steger, J. L., "Numerical Solution of the Azimuthal-Invariant Thin-Layer Navier-Stokes Equations," AIAA Paper 79-19475, Jan. 1979.

2. Nietubicz, C. J., Pulliam, T. H., and Steger, J. L., "Numerical Solution of the Azimuthal-Invariant Thin-Layer Navier-Stokes Equations," AIAA Journal, Vol. 18, Dec. 1980, pp. 1411-1412.

3. Pulliam, T. H., and Steger, J. L., "On Implicit FiniteDifference Simulations of Three-Dimensional Flow," AIAA Paper 78-10, Jan. 1978.

4. Anderson, D. A., Tannehill, J. C., and Pletcher, R. H., Computational Fluid Mechanics and Heat Transfer. New York: McGraw-Hill, Inc., 1984, pp. 545546.

5. Baldwin, B. S., and Lomax, H., "Thin-Layer Approximation and Algebraic Model for Separated Turbulent Flows," AIAA Paper 78-257, Jan. 1978.

6. Chima, R. V., Giel, P. W., and Boyle, R. J., "An Algebraic Turbulence Model for Three-Dimensional Viscous Flows," NASA TM-105931, Jan. 1993.

7. Chima, Rodrick V., "Explicit Multigrid Algorithm for Quasi-Three-Dimensional Viscous Flows in Turbomachinery," AIAA Journal of Propulsion and Power, Vol. 3, No. 5, Sept.-Oct. 1987, pp. 397-405. 
8. Chima, Rodrick V., and Yokota, Jeffrey W., "Numerical Analysis of Three-Dimensional Viscous Internal Flows," AIAA Journal, Vol. 28, No. 5, May 1990, pp. 798-806 (also NASA TM-100878).

9. Chima, Rodrick V., "Viscous Three-Dimensional Calculations of Transonic Fan Performance," in CFD Techniques for Propulsion Applications, AGARD Conference Proceedings No. CP-510, AGARD, Neuilly-Sur-Seine, France, Feb. 1992, pp. 21-1 to 21-19 (also NASA TM-103800).

10. Jameson, A., Schmidt, W., and Turkel, E., "Numerical Solutions of the Euler Equations by Finite Volume Methods Using Runge-Kutta Time-Stepping Schemes," AIAA Paper 81-1259, June 1981.

11. Kunz, R. F., and Lakshminarayana, B., "Computation of Supersonic and Low Subsonic Cascade Flows Using an Explicit Navier-Stokes Technique and the $K$ - $\varepsilon$ Turbulence Model," in Computational Fluid Dynamics Symposium on Aeropropulsion, NASA CP-10045, Apr. 1990.

12. Martinelli, L., and Jameson, A., "Validation of a Multigrid Method for the Reynolds Averaged Equations," AIAA Paper 88-0414, Jan. 1988.

13. Arnone, A., "Viscous Analysis of Three-Dimensional Rotor Flows Using a Multigxid Method," NASA TM-106266 (ICOMP-93-25), July 1993.

14. Jameson, A., and Baker, T. J., "Solution of the Euler Equations for Complex Configurations," AIAA Paper 83-1929, July 1983.

15. Miller, D. P., "TIGGERC - Turbomachinery Interactive Grid Generator for 2-D Grid Applications and Users Guide," NASA TM-106586, May 1994.

16. Steger, J. L., and Sorenson, R. L., "Automatic MeshPoint Clustering Near a Boundary in Grid Generation with Elliptic Partial Differential Equations," $J$. Comp. Phys., Vol. 33, No. 3, Dec. 1979, pp. 405-410.

17. Schlichting, Hermann, Boundary-Layer Theory. McGraw-Hill, Inc., New York, 1968, p. 674.

18. Winter, K. G., Rotta, J. C., and Smith, K. G., "Studies of the Turbulent Boundary Layer on a Waisted Body of Revolution in Subsonic and Supersonic Flow," ARC R\&M No. 3633, 1970.

19. Mateer, G. G., Brosh, A., and Viegas, J. R., "A Normal Shock-Wave Turbulent Boundary-Layer Interaction at Transonic Speeds," AIAA Paper 76-161, Jan. 1976.

20. Viegas, J. R., and Horstman, C. C., "Comparison of Multiequation Turbulence Models for Several Shock Boundary-Layer Interaction Flows," AIAA Journal, Vol. 17, Aug. 1979, pp. 811-820.
21. Kline, S. J., Cantwell, B. J., and Lilley, G. M., 198081 AFOSR-HTTM -Stanford Conference on Complex Turbulent Flows: Comparison of Computation and Experiment. Stanford University, Stanford, California, Vol. 1, 1981, pp. 510-518.

22. White, Frank M., Viscous Fluid Flow. McGraw-Hill, Inc., New York, 1974, p. 475.

23. White, Frank M., Viscous Fluid Flow. McGraw-Hill, Inc., New York, 1974, p. 581.

24. Wilcox, David C., Turbulence Modeling for CFD. DCW Industries, Inc., 1994.

25. Seddon, J., "The Flow Produced by Interaction of a Turbulent Boundary Layer With a Normal Shock Wave of Strength Sufficient to Cause Separation," ARC R\&M No. 3502, 1960.

26. East, L. F., "The Application of a Laser Anemometer to the Investigation of Shock Wave/Boundary Layer Interactions," AGARD-CP-193 (also RAE Technical Memo AERO 1666), Feb. 1976.

27. Delery, J., and Marvin, J.G., "Shock-Wave Boundary Layer Interactions," AGARDograph 280, 1986.

28. Keller, Jacob J., "On the Practical Application of Vortex Breakdown Theory to Axially Symmetrical and Three-Dimensional Compressible Flows," Physics of Fluids, Vol. 6, No. 4, April 1994, p. 1522.

29. Benjamin, T. B., "Theory of the Vortex Breakdown Phenomenon," Journal of Fluid Mechanics, Vol. 14, 1962, pp. 593-629.

30. Liebovich, S., "The Structure of Vortex Breakdown," Ann. Rev. Fluid Mech., 10, 1978.

31. Escudier, M. P., "Vortex Breakdown: Observations and Explanations," Prog. Aerospace Sci., Vol. 25, 1988, pp. 189-229.

32. Chima, R. V., "A k- $\omega$ Turbulence Model for QuasiThree-Dimensional Turbomachinery Flows," AIAA Paper 96-0248 (also NASA TM-107051), Jan. 1996.

33. Wilcox, David C., "Simulation of Transition with a Two-Equation Turbulence Model," AIAA Journal, Vol. 32, No. 2, Feb. 1994, pp. 247-255.

34. Turkel, E., "Preconditioning Methods for Solving the Incompressible and Low Speed Compressible Equations," Journal of Computational Physics, Vol. 72, 1987, pp. 277-298.

35. Turkel, E., "A Review of Preconditioning Methods for Fluid Dynamics," Applied Numerical Mathematics, Vol. 12, 1993, pp. 257-284.

36. Choi, Y.-H. and Merkle, C. L., "The Application of Preconditioning to Viscous Flows," Journal of Computational Physics, Vol. 105, 1993, pp. 207-223. 
Public reporting burden for this collection of information is estimated to average 1 hour per response, including the time for reviewing instructions, searching existing data sources, gathering and maintaining the data needed, and completing and reviewing the collection of information. Send comments regarding this burden estimate or any other aspect of this collection of information, including suggestions for reducing this burden, to Washington Headquarters Services, Directorate for Intormation Operations and Reports, 1215 Jefierson Davis Highway, Suite 1204, Arlington, VA 22202-4302, and to the Offico of Management and Budget, Papenwork Reduction Project (0704-0188). Washington, DC 20503.

\begin{tabular}{|l|l|l|}
\hline 1. AGENCY USE ONLY (Leave blank) & $\begin{array}{c}\text { 2. REPORT DATE } \\
\text { November } 1995\end{array}$ & $\begin{array}{r}\text { 3. REPORT TYPE AND DATES COVERED } \\
\text { Technical Memorandum }\end{array}$ \\
\hline
\end{tabular}

4. TITLE AND SUBTITLE

5. FUNDING NUMBERS

Rapid Numerical Simulation of Viscous Axisymmetric Flow Fields

6. AUTHOR(S)

WU-505-62-10

Daniel L. Tweedt and Rodrick V. Chima

7. PERFORMING ORGANIZATION NAME(S) AND ADDRESS(ES)

8. PERFORMING ORGANIZATION REPORT NUMBER

National Aeronautics and Space Administration

Lewis Research Center

Cleveland, Ohio 44135-3191

E-10001

9. SPONSORING/MONITORING AGENCY NAME(S) AND ADDRESS(ES)

10. SPONSORINGMONITORING AGENCY REPORT NUMBER

National Aeronautics and Space Administration

Washington, D.C. 20546-0001

NASA TM-107103

AIAA-96-0449

11. SUPPLEMENTARY NOTES

Prepared for the 34th Aerospace Sciences Meeting and Exhibit sponsored by the American Institute of Aeronautics and Astronautics, Reno, Nevada, January 15-18, 1996. Responsible person, Daniel L. Tweedt, organization code 2760, (216) 433-3592.

12a. DISTRIBUTIONAVAILABILTYY STATEMENT

12b. DISTRIBUTION CODE

Unclassified - Unlimited

Subject Category 07

This publication is available from the NASA Center for Aerospace Information, (301) 621-0390.

13. ABSTRACT (Maximum 200 words)

A two-dimensional Navier-Stokes code has been developed for rapid numerical simulation of axisymmetric flow fields, including flow fields with an azimuthal velocity component. The azimuthal-invariant Navier-Stokes equations in a cylindrical coordinate system are mapped to a general body-fitted coordinate system, with the streamwise viscous terms then neglected by applying the thin-layer approximation. Turbulence effects are modeled using an algebraic model, typically the Baldwin-Lomax turbulence model, although a modified Cebeci-Smith model can also be used. The equations are discretized using central finite differences and solved using a multistage Runge-Kutta algorithm with a spatiallyvarying time step and implicit residual smoothing. Results are presented for calculations of supersonic flow over a waisted body-of-revolution, transonic flow through a normal shock wave in a straight circular duct of constant crosssectional area, swirling supersonic (inviscid) flow through a strong shock in a straight radial duct, and swirling subsonic flow in an annular-to-circular diffuser duct. Comparisons between computed and experimental results are in fair to good agreement, demonstrating that the viscous code can be a useful tool for practical engineering design and analysis work.

14. SUBJECT TERMS

Axisymmetric; Azimuthal-invariant; Computational fluid dynamics; Viscous flow; Navier-Stokes equations; Multistage Runge-Kutta scheme

\begin{tabular}{|c|c|c|}
\hline $\begin{array}{c}\text { 17. SECURTY CLASSIFICATION } \\
\text { OF REPORT } \\
\text { Unclassified }\end{array}$ & $\begin{array}{c}\text { 18. SECURTY CLASSIFICATION } \\
\text { OF THIS PAGE } \\
\text { Unclassified }\end{array}$ & $\begin{array}{c}\text { 19. SECURITY CLASSIFICATION } \\
\text { OF ABSTRACT } \\
\text { Unclassified }\end{array}$ \\
\hline
\end{tabular}

